\title{
Vector-Valued Inequalities in the Morrey Type Spaces
}

\author{
Hua Wang \\ College of Mathematics and Econometrics, Hunan University, Changsha 410082, China \\ Correspondence should be addressed to Hua Wang; wanghua@pku.edu.cn \\ Received 8 February 2014; Accepted 3 May 2014; Published 15 May 2014 \\ Academic Editor: Ingo Witt
}

Copyright (C) 2014 Hua Wang. This is an open access article distributed under the Creative Commons Attribution License, which permits unrestricted use, distribution, and reproduction in any medium, provided the original work is properly cited.

We will obtain the strong type and weak type estimates for vector-valued analogues of classical Hardy-Littlewood maximal function, weighted maximal function, and singular integral operators in the weighted Morrey spaces $L^{p, \kappa}(w)$ when $1 \leq p<\infty$ and $0<\kappa<1$, and in the generalized Morrey spaces $L^{p, \Phi}$ for $1 \leq p<\infty$, where $\Phi$ is a growth function on $(0, \infty)$ satisfying the doubling condition.

\section{Introduction}

The classical Hardy-Littlewood maximal function $M f(x)$ is defined for a locally integrable function $f$ on $\mathbb{R}^{n}$ by

$$
M f(x)=\sup _{x \in B} \frac{1}{|B|} \int_{B}|f(y)| d y
$$

where the supremum is taken over all balls $B$ containing $x$. It is well known that the maximal operator $M$ maps $L^{p}\left(\mathbb{R}^{n}\right)$ into $L^{p}\left(\mathbb{R}^{n}\right)$ for all $1<p \leq \infty$ and $L^{1}\left(\mathbb{R}^{n}\right)$ into $W L^{1}\left(\mathbb{R}^{n}\right)$. Let $\vec{f}=\left(f_{1}, f_{2}, \ldots\right)$ be a sequence of locally integrable functions on $\mathbb{R}^{n}$. For any $x \in \mathbb{R}^{n}$, we define $M(\vec{f})=\left(M\left(f_{1}\right), M\left(f_{2}\right), \ldots\right)$ and

$$
\mathbf{M}_{q}(\vec{f})(x)=\left(\sum_{j}\left|M\left(f_{j}\right)(x)\right|^{q}\right)^{1 / q}, \quad 1<q<\infty .
$$

This nonlinear operator was introduced by Fefferman and Stein in [1], and since then it has played an important role in the development of modern harmonic analysis. In this remarkable paper [1], Fefferman and Stein extended the classical maximal theorem to the case of vector-valued functions.
Theorem 1 (see [1]). Let $1<p<\infty$. Then, for every $1<$ $q<\infty$, there exists a constant $C>0$ independent of $\vec{f}=$ $\left(f_{1}, f_{2}, \ldots\right)$ such that

$$
\left\|\left(\sum_{j}\left|M\left(f_{j}\right)\right|^{q}\right)^{1 / q}\right\|_{L^{p}} \leq C\left\|\left(\sum_{j}\left|f_{j}\right|^{q}\right)^{1 / q}\right\|_{L^{p}} .
$$

Theorem 2 (see [1]). Let $p=1$. Then, for every $1<q<\infty$, there exists a constant $C>0$ independent of $\vec{f}=\left(f_{1}, f_{2}, \ldots\right)$ such that

$$
\left\|\left(\sum_{j}\left|M\left(f_{j}\right)\right|^{q}\right)^{1 / q}\right\|_{W L^{1}} \leq C\left\|\left(\sum_{j}\left|f_{j}\right|^{q}\right)^{1 / q}\right\|_{L^{1}} .
$$

A weight $w$ is a nonnegative, locally integrable function on $\mathbb{R}^{n} ; B=B\left(x_{0}, r_{B}\right)$ denotes the ball with the center $x_{0}$ and radius $r_{B}$. For $1<p<\infty$, a weight function $w$ is said to belong to $A_{p}$, if there is a constant $C>0$ such that, for every ball $B \subseteq \mathbb{R}^{n}$ (see $\left.[2,3]\right)$,

$$
\left(\frac{1}{|B|} \int_{B} w(x) d x\right)\left(\frac{1}{|B|} \int_{B} w(x)^{-1 /(p-1)} d x\right)^{p-1} \leq C .
$$

For the case $p=1, w \in A_{1}$, if there is a constant $C>0$ such that, for every ball $B \subseteq \mathbb{R}^{n}$,

$$
\frac{1}{|B|} \int_{B} w(x) d x \leq C \cdot \underset{x \in B}{\operatorname{essinf}} w(x) .
$$


A weight function $w \in A_{\infty}$ if it satisfies the $A_{p}$ condition for some $1 \leq p<\infty$. We say that $w \in \Delta_{2}$, if for any ball $B$ there exists an absolute constant $C>0$ such that

$$
w(2 B) \leq C w(B) .
$$

It is well known that if $w \in A_{p}$ with $1 \leq p<\infty$, then $w \in \Delta_{2}$. Moreover, if $w \in A_{\infty}$, then, for all balls $B$ and all measurable subsets $E$ of $B$, there exists $\delta>0$ such that

$$
\frac{w(E)}{w(B)} \leq C\left(\frac{|E|}{|B|}\right)^{\delta}
$$

Given a ball $B$ and $\lambda>0, \lambda B$ denotes the ball with the same center as $B$ whose radius is $\lambda$ times that of $B$. For a given weight function $w$ and a measurable set $E$, we also denote the Lebesgue measure of $E$ by $|E|$ and the weighted measure of $E$ by $w(E)$, where $w(E)=\int_{E} w(x) d x$.

Given a weight function $w$ on $\mathbb{R}^{n}$, for $1 \leq p<\infty$, the weighted Lebesgue space $L_{w}^{p}\left(\mathbb{R}^{n}\right)$ is defined as the set of all functions $f$ such that

$$
\|f\|_{L_{w}^{p}}=\left(\int_{\mathbb{R}^{n}}|f(x)|^{p} w(x) d x\right)^{1 / p}<\infty .
$$

We also denote by $W L_{w}^{1}\left(\mathbb{R}^{n}\right)$ the weighted weak space consisting of all measurable functions $f$ such that

$$
\|f\|_{W L_{w}^{1}}=\sup _{\lambda>0} \lambda \cdot w\left(\left\{x \in \mathbb{R}^{n}:|f(x)|>\lambda\right\}\right)<\infty .
$$

In particular, when $w$ equals to a constant function, we will denote $L_{w}^{p}\left(\mathbb{R}^{n}\right)$ and $W L_{w}^{1}\left(\mathbb{R}^{n}\right)$ simply by $L^{p}\left(\mathbb{R}^{n}\right)$ and $W L^{1}\left(\mathbb{R}^{n}\right)$.

In [4], Andersen and John considered the weighted version of Fefferman-Stein maximal inequality and showed the following.

Theorem 3 (see [4]). Let $1<p<\infty$ and $w \in A_{p}$. Then, for every $1<q<\infty$, there exists a constant $C>0$ independent of $\vec{f}=\left(f_{1}, f_{2}, \ldots\right)$ such that

$$
\left\|\left(\sum_{j}\left|M\left(f_{j}\right)\right|^{q}\right)^{1 / q}\right\|_{L_{w}^{p}} \leq C\left\|\left(\sum_{j}\left|f_{j}\right|^{q}\right)^{1 / q}\right\|_{L_{w}^{p}} .
$$

Theorem 4 (see [4]). Let $p=1$ and $w \in A_{1}$. Then, for every $1<q<\infty$, there exists a constant $C>0$ independent of $\vec{f}=\left(f_{1}, f_{2}, \ldots\right)$ such that

$$
\left\|\left(\sum_{j}\left|M\left(f_{j}\right)\right|^{q}\right)^{1 / q}\right\|_{W L_{w}^{1}} \leq C\left\|\left(\sum_{j}\left|f_{j}\right|^{q}\right)^{1 / q}\right\|_{L_{w}^{1}} .
$$

Given a weight $w$, the weighted maximal function is defined as

$$
M_{w} f(x)=\sup _{x \in B} \frac{1}{w(B)} \int_{B}|f(y)| w(y) d y
$$

where the supremum is taken over all balls $B$ containing $x$. One says that $u \in A_{p}(w)$ with $1<p<\infty$, if $u, w \in \Delta_{2}$ and there is a constant $C>0$ such that, for every ball $B \subseteq \mathbb{R}^{n}$,

$$
\frac{u(B)}{w(B)}\left(\frac{1}{w(B)} \int_{B}\left(\frac{w(x)}{u(x)}\right)^{1 /(p-1)} w(x) d x\right)^{p-1} \leq C .
$$

If $\vec{f}=\left(f_{1}, f_{2}, \ldots\right)$ is a sequence of locally integrable functions on $\mathbb{R}^{n}$, then, for any $x \in \mathbb{R}^{n}$, we can also define $M_{w}(\vec{f})=$ $\left(M_{w}\left(f_{1}\right), M_{w}\left(f_{2}\right), \ldots\right)$ and

$$
\mathbf{M}_{w, q}(\vec{f})(x)=\left(\sum_{j}\left|M_{w}\left(f_{j}\right)(x)\right|^{q}\right)^{1 / q}, \quad 1<q<\infty .
$$

In [5], Wu proved the following theorem.

Theorem 5 (see [5]). Let $1<p<\infty$ and $u \in A_{p}(w)$. Then, for every $1<q<\infty$, there exists a constant $C>0$ independent of $\vec{f}=\left(f_{1}, f_{2}, \ldots\right)$ such that

$$
\left\|\left(\sum_{j}\left|M_{w}\left(f_{j}\right)\right|^{q}\right)^{1 / q}\right\|_{L_{u}^{p}} \leq C\left\|\left(\sum_{j}\left|f_{j}\right|^{q}\right)^{1 / q}\right\|_{L_{u}^{p}} .
$$

Obviously, by the definition, we have $w \in A_{p}(w)$ with $1<p<\infty$, if $w \in \Delta_{2}$. Thus, as a direct consequence of Theorem 5, we get the following theorem.

Theorem $5^{\prime}$. Let $1<p<\infty$ and $w \in \Delta_{2}$. Then, for every $1<q<\infty$, there exists a constant $C>0$ independent of $\vec{f}=\left(f_{1}, f_{2}, \ldots\right)$ such that

$$
\left\|\left(\sum_{j}\left|M_{w}\left(f_{j}\right)\right|^{q}\right)^{1 / q}\right\|_{L_{w}^{p}} \leq C\left\|\left(\sum_{j}\left|f_{j}\right|^{q}\right)^{1 / q}\right\|_{L_{w}^{p}} .
$$

Let us now consider the vector-valued singular integral operators. Suppose that $S^{n-1}$ is the unit sphere in $\mathbb{R}^{n}(n \geq 2)$ equipped with the normalized Lebesgue measure $d \sigma$. Let $\Omega \epsilon$ $L^{s}\left(S^{n-1}\right)$ with $1<s \leq \infty$ be homogeneous of degree zero and satisfy the cancellation condition

$$
\int_{S^{n-1}} \Omega\left(x^{\prime}\right) d \sigma\left(x^{\prime}\right)=0
$$

where $x^{\prime}=x /|x|$ for any $x \neq 0$. Then, the homogeneous singular integral operator $T_{\Omega}$ is defined by

$$
T_{\Omega} f(x)=\text { P.V. } \int_{\mathbb{R}^{n}} \frac{\Omega(x-y)}{|x-y|^{n}} f(y) d y .
$$

For $1<s \leq \infty$, we say that $\Omega$ satisfies the $L^{s}$-Dini condition and write $\Omega \in\left(D_{s}\right)$, if $\Omega \in L^{s}\left(S^{n-1}\right)$ and

$$
\int_{0}^{1} \frac{\omega_{s}(\delta)}{\delta} d \delta<\infty
$$


where $\omega_{s}(\delta)$ denotes the integral modulus of continuity of order $s$ of $\Omega$ defined by

$$
\omega_{s}(\delta):=\sup _{|\rho|<\delta}\left(\int_{S^{n-1}}\left|\Omega\left(\rho x^{\prime}\right)-\Omega\left(x^{\prime}\right)\right|^{s} d \sigma\left(x^{\prime}\right)\right)^{1 / s},
$$

$\rho$ is a rotation in $\mathbb{R}^{n}$, and $|\rho|=\sup _{z^{\prime} \in S^{n-1}}\left|\rho z^{\prime}-z^{\prime}\right|$. When $s=\infty, \omega_{s}(\delta)$ is defined by the following expression:

$$
\omega_{\infty}(\delta)=\sup _{\left|x^{\prime}-y^{\prime}\right|<\delta}\left\{\left|\Omega\left(x^{\prime}\right)-\Omega\left(y^{\prime}\right)\right|: x^{\prime}, y^{\prime} \in S^{n-1}\right\} .
$$

Let $\vec{f}=\left(f_{1}, f_{2}, \ldots\right)$ be a sequence of locally integrable functions on $\mathbb{R}^{n}$. For any $x \in \mathbb{R}^{n}$, as above, the vector-valued singular integral operators with kernels $\Omega \in\left(D_{s}\right)$ can be defined as $T_{\Omega}(\vec{f})=\left(T_{\Omega}\left(f_{1}\right), T_{\Omega}\left(f_{2}\right), \ldots\right)$ and

$$
\mathbf{T}_{\Omega, q}(\vec{f})(x)=\left(\sum_{j}\left|T_{\Omega}\left(f_{j}\right)(x)\right|^{q}\right)^{1 / q}, \quad 1<q<\infty .
$$

In [4], Andersen and John derived the weighted strong and weak type estimates for vector-valued singular integral operators with kernels $\Omega \in\left(D_{\infty}\right)$ (see also [6,7]).

Theorem 6 (see [4]). Let $T_{\Omega}$ be a singular integral operator with kernel $\Omega \in\left(D_{\infty}\right)$. If $1<p<\infty$ and $w \in A_{p}$, then, for every $1<q<\infty$, there exists a constant $C>0$ independent of $\vec{f}=\left(f_{1}, f_{2}, \ldots\right)$ such that

$$
\left\|\left(\sum_{j}\left|T_{\Omega}\left(f_{j}\right)\right|^{q}\right)^{1 / q}\right\|_{L_{w}^{p}} \leq C\left\|\left(\sum_{j}\left|f_{j}\right|^{q}\right)^{1 / q}\right\|_{L_{w}^{p}} .
$$

Theorem 7 (see [4]). Let $T_{\Omega}$ be a singular integral operator with kernel $\Omega \in\left(D_{\infty}\right)$. If $p=1$ and $w \in A_{1}$, then, for every $1<q<\infty$, there exists a constant $C>0$ independent of $\vec{f}=\left(f_{1}, f_{2}, \ldots\right)$ such that

$$
\left\|\left(\sum_{j}\left|T_{\Omega}\left(f_{j}\right)\right|^{q}\right)^{1 / q}\right\|_{W L_{w}^{1}} \leq C\left\|\left(\sum_{j}\left|f_{j}\right|^{q}\right)^{1 / q}\right\|_{L_{w}^{1}} .
$$
in [7].

Theorem 6 was later extended by Rubio de Francia et al.

Theorem 8 (see [7]). Let $1<s<\infty$ and let $T_{\Omega}$ be a singular integral operator with kernel $\Omega \in\left(D_{s}\right)$. If $s^{\prime} \leq p<\infty$ and $w \in A_{p / s^{\prime}}$, then, for every $1<q<\infty$, there exists a constant $C>0$ independent of $\vec{f}=\left(f_{1}, f_{2}, \ldots\right)$ such that

$$
\left\|\left(\sum_{j}\left|T_{\Omega}\left(f_{j}\right)\right|^{q}\right)^{1 / q}\right\|_{L_{w}^{p}} \leq C\left\|\left(\sum_{j}\left|f_{j}\right|^{q}\right)^{1 / q}\right\|_{L_{w}^{p}} .
$$

In particular, if we take $w$ to be a constant function, then we immediately get the following unweighted results (see also $[8])$.
Theorem 9. Let $T_{\Omega}$ be a singular integral operator with kernel $\Omega \in\left(D_{\infty}\right)$ and $1<p<\infty$. Then, for every $1<q<\infty$, there exists a constant $C>0$ independent of $\vec{f}=\left(f_{1}, f_{2}, \ldots\right)$ such that

$$
\left\|\left(\sum_{j}\left|T_{\Omega}\left(f_{j}\right)\right|^{q}\right)^{1 / q}\right\|_{L^{p}} \leq C\left\|\left(\sum_{j}\left|f_{j}\right|^{q}\right)^{1 / q}\right\|_{L^{p}} .
$$

Theorem 10. Let $T_{\Omega}$ be a singular integral operator with kernel $\Omega \in\left(D_{\infty}\right)$ and $p=1$. Then, for every $1<q<\infty$, there exists a constant $C>0$ independent of $\vec{f}=\left(f_{1}, f_{2}, \ldots\right)$ such that

$$
\left\|\left(\sum_{j}\left|T_{\Omega}\left(f_{j}\right)\right|^{q}\right)^{1 / q}\right\|_{W L^{1}} \leq C\left\|\left(\sum_{j}\left|f_{j}\right|^{q}\right)^{1 / q}\right\|_{L^{1}} .
$$

Theorem 11. Let $1<s<\infty$ and let $T_{\Omega}$ be a singular integral operator with kernel $\Omega \in\left(D_{s}\right)$. If $s^{\prime} \leq p<\infty$, then, for every $1<q<\infty$, there exists a constant $C>0$ independent of $\vec{f}=\left(f_{1}, f_{2}, \ldots\right)$ such that

$$
\left\|\left(\sum_{j}\left|T_{\Omega}\left(f_{j}\right)\right|^{q}\right)^{1 / q}\right\|_{L^{p}} \leq C\left\|\left(\sum_{j}\left|f_{j}\right|^{q}\right)^{1 / q}\right\|_{L^{p}} .
$$

\section{Our Main Results}

The classical Morrey spaces $\mathscr{L}^{p, \lambda}$ were originally introduced by Morrey in [9] to study the local behavior of solutions to second order elliptic partial differential equations. For the boundedness of the Hardy-Littlewood maximal operator, the fractional integral operator, and the Calderón-Zygmund singular integral operator on these spaces, we refer the reader to [10-12]. In [13], Mizuhara introduced the generalized Morrey spaces $L^{p, \Phi}$ which were later extended and studied by many authors (see [14-18]). In [19], Komori and Shirai defined the weighted Morrey spaces $L^{p, \kappa}(w)$ which could be viewed as an extension of weighted Lebesgue spaces and then studied the boundedness of the above classical operators in harmonic analysis on these weighted spaces.

Definition A (see [19]). Let $1 \leq p<\infty$, let $0<\kappa<1$, and let $w$ be a weight function on $\mathbb{R}^{n}$. Then, the weighted Morrey space is defined by

$$
L^{p, \kappa}(w)=\left\{f \in L_{\mathrm{loc}}^{p}(w):\|f\|_{L^{p, \kappa}(w)}<\infty\right\},
$$

where

$$
\|f\|_{L^{p, \kappa}(w)}=\sup _{B}\left(\frac{1}{w(B)^{\kappa}} \int_{B}|f(x)|^{p} w(x) d x\right)^{1 / p}
$$

and the supremum is taken over all balls $B$ in $\mathbb{R}^{n}$. 
For $p=1$ and $0<\kappa<1$, we also denote by $W L^{1, \kappa}(w)$ the weighted weak Morrey spaces of all measurable functions $f$ satisfying

$$
\begin{aligned}
\|f\|_{W L^{1, \kappa}(w)} & =\sup _{B} \sup _{\lambda>0} \frac{1}{w(B)^{\kappa}} \lambda \cdot w(\{x \in B:|f(x)|>\lambda\}) \\
& <\infty .
\end{aligned}
$$

Let $\Phi=\Phi(r), r>0$, be a growth function, that is, a positive increasing function in $(0, \infty)$, and satisfy the following doubling condition:

$$
\Phi(2 r) \leq D \cdot \Phi(r), \quad \forall r>0
$$

where $D=D(\Phi) \geq 1$ is a doubling constant independent of $r$.

Definition $B$ (see [13]). Let $1 \leq p<\infty$. One denotes by $L^{p, \Phi}=L^{p, \Phi}\left(\mathbb{R}^{n}\right)$ the space of all locally integrable functions $f$ defined on $\mathbb{R}^{n}$, such that for every $x_{0} \in \mathbb{R}^{n}$ and all $r>0$

$$
\int_{B\left(x_{0}, r\right)}|f(x)|^{p} d x \leq C^{p} \cdot \Phi(r)
$$

where $B\left(x_{0}, r\right)=\left\{x \in \mathbb{R}^{n}:\left|x-x_{0}\right|<r\right\}$ is the ball centered at $x_{0}$ and with radius $r>0$. Then, one lets $\|f\|_{L^{p, \Phi}}$ be the smallest constant $C>0$ satisfying (34) and $L^{p, \Phi}\left(\mathbb{R}^{n}\right)$ becomes a Banach space with norm $\|\cdot\|_{L^{p, \Phi}}$.

Obviously, when $\Phi(r)=r^{\lambda}$ with $0<\lambda<n, L^{p, \Phi}$ is just the classical Morrey spaces introduced in [9]. We also denote by $W L^{1, \Phi}=W L^{1, \Phi}\left(\mathbb{R}^{n}\right)$ the generalized weak Morrey spaces of all measurable functions $f$ for which

$$
\sup _{\lambda>0} \lambda \cdot\left|\left\{x \in B\left(x_{0}, r\right):|f(x)|>\lambda\right\}\right| \leq C \cdot \Phi(r),
$$

for every $x_{0} \in \mathbb{R}^{n}$ and all $r>0$. The smallest constant $C>0$ satisfying (35) is also denoted by $\|f\|_{W L^{1, \Phi}}$.

Let $\vec{f}=\left(f_{1}, f_{2}, \ldots\right)$ be a sequence of locally integrable functions on $\mathbb{R}^{n}$. Now, let us formulate our main results as follows. For the continuity properties of vector-valued HardyLittlewood maximal function, weighted maximal function, and singular integral operators in the weighted Morrey spaces $L^{p, \kappa}(w)$ for all $1 \leq p<\infty$ and $0<\kappa<1$, we will prove the following.

Theorem 12. Let $1<p<\infty, 0<\kappa<1$, and $w \in A_{p}$. Then, for all $1<q<\infty$, there exists a constant $C>0$ independent of $\vec{f}=\left(f_{1}, f_{2}, \ldots\right)$ such that

$$
\left\|\left(\sum_{j}\left|M\left(f_{j}\right)\right|^{q}\right)^{1 / q}\right\|_{L^{p, \kappa}(w)} \leq C\left\|\left(\sum_{j}\left|f_{j}\right|^{q}\right)^{1 / q}\right\|_{L^{p, \kappa}(w)} .
$$

Theorem 13. Let $p=1,0<\kappa<1$, and $w \in A_{1}$. Then, for all $1<q<\infty$, there exists a constant $C>0$ independent of $\vec{f}=\left(f_{1}, f_{2}, \ldots\right)$ such that

$$
\left\|\left(\sum_{j}\left|M\left(f_{j}\right)\right|^{q}\right)^{1 / q}\right\|_{W L^{1, \kappa}(w)} \leq C\left\|\left(\sum_{j}\left|f_{j}\right|^{q}\right)^{1 / q}\right\|_{L^{1, \kappa}(w)} .
$$

Theorem 14. Let $1<p<\infty, 0<\kappa<1$, and $w \in \Delta_{2}$. Then, for all $1<q<\infty$, there exists a constant $C>0$ independent of $\vec{f}=\left(f_{1}, f_{2}, \ldots\right)$ such that

$$
\left\|\left(\sum_{j}\left|M_{w}\left(f_{j}\right)\right|^{q}\right)^{1 / q}\right\|_{L^{p, \kappa}(w)} \leq C\left\|\left(\sum_{j}\left|f_{j}\right|^{q}\right)^{1 / q}\right\|_{L^{p, \kappa}(w)} .
$$

Theorem 15. Let $T_{\Omega}$ be a singular integral operator with kernel $\Omega \in\left(D_{\infty}\right)$. If $1<p<\infty, 0<\kappa<1$, and $w \in A_{p}$, then, for all $1<q<\infty$, there exists a constant $C>0$ independent of $\vec{f}=\left(f_{1}, f_{2}, \ldots\right)$ such that

$$
\left\|\left(\sum_{j}\left|T_{\Omega}\left(f_{j}\right)\right|^{q}\right)^{1 / q}\right\|_{L^{p, \kappa}(w)} \leq C\left\|\left(\sum_{j}\left|f_{j}\right|^{q}\right)^{1 / q}\right\|_{L^{p, \kappa}(w)} .
$$

Theorem 16. Let $T_{\Omega}$ be a singular integral operator with kernel $\Omega \in\left(D_{\infty}\right)$. If $p=1,0<\kappa<1$, and $w \in A_{1}$, then, for all $1<q<\infty$, there exists a constant $C>0$ independent of $\vec{f}=\left(f_{1}, f_{2}, \ldots\right)$ such that

$$
\left\|\left(\sum_{j}\left|T_{\Omega}\left(f_{j}\right)\right|^{q}\right)^{1 / q}\right\|_{W L^{1, \kappa}(w)} \leq C\left\|\left(\sum_{j}\left|f_{j}\right|^{q}\right)^{1 / q}\right\|_{L^{1, \kappa}(w)} .
$$

Theorem 17. Let $1<s<\infty$ and let $T_{\Omega}$ be a singular integral operator with kernel $\Omega \in\left(D_{s}\right)$. If $s^{\prime} \leq p<\infty, 0<\kappa<1$, and $w \in A_{p / s^{\prime}}$, then, for all $1<q<\infty$, there exists a constant $C>0$ independent of $\vec{f}=\left(f_{1}, f_{2}, \ldots\right)$ such that

$$
\left\|\left(\sum_{j}\left|T_{\Omega}\left(f_{j}\right)\right|^{q}\right)^{1 / q}\right\|_{L^{p, \kappa}(w)} \leq C\left\|\left(\sum_{j}\left|f_{j}\right|^{q}\right)^{1 / q}\right\|_{L^{p, \kappa}(w)} .
$$

For the vector-valued inequalities in the generalized Morrey spaces $L^{p, \Phi}$ for all $1 \leq p<\infty$, we will show the following.

Theorem 18. Assume that $\Phi$ satisfies (33) and $1 \leq D(\Phi)<2^{n}$. Then, for all $1<p \leq q<\infty$, there exists a constant $C>0$ independent of $\vec{f}=\left(f_{1}, f_{2}, \ldots\right)$ such that

$$
\left\|\left(\sum_{j}\left|M\left(f_{j}\right)\right|^{q}\right)^{1 / q}\right\|_{L^{p, \Phi}} \leq C\left\|\left(\sum_{j}\left|f_{j}\right|^{q}\right)^{1 / q}\right\|_{L^{p, \Phi}} .
$$


Theorem 19. Assume that $\Phi$ satisfies (33) and $1 \leq D(\Phi)<2^{n}$. Then, for $p=1$ and all $1<q<\infty$, there exists a constant $C>0$ independent of $\vec{f}=\left(f_{1}, f_{2}, \ldots\right)$ such that

$$
\left\|\left(\sum_{j}\left|M\left(f_{j}\right)\right|^{q}\right)^{1 / q}\right\|_{W L^{1, \Phi}} \leq C\left\|\left(\sum_{j}\left|f_{j}\right|^{q}\right)^{1 / q}\right\|_{L^{1, \Phi}} .
$$

Theorem 20. Let $T_{\Omega}$ be a singular integral operator with kernel $\Omega \in\left(D_{\infty}\right)$. Assume that $\Phi$ satisfies (33) and $1 \leq D(\Phi)<$ $2^{n}$; then, for all $1<p, q<\infty$, there exists a constant $C>0$ independent of $\vec{f}=\left(f_{1}, f_{2}, \ldots\right)$ such that

$$
\left\|\left(\sum_{j}\left|T_{\Omega}\left(f_{j}\right)\right|^{q}\right)^{1 / q}\right\|_{L^{p, \Phi}} \leq C\left\|\left(\sum_{j}\left|f_{j}\right|^{q}\right)^{1 / q}\right\|_{L^{p, \Phi}} .
$$

Theorem 21. Let $T_{\Omega}$ be a singular integral operator with kernel $\Omega \in\left(D_{\infty}\right)$. Assume that $\Phi$ satisfies (33) and $1 \leq D(\Phi)<2^{n}$; then, for $p=1$ and all $1<q<\infty$, there exists a constant $C>0$ independent of $\vec{f}=\left(f_{1}, f_{2}, \ldots\right)$ such that

$$
\left\|\left(\sum_{j}\left|T_{\Omega}\left(f_{j}\right)\right|^{q}\right)^{1 / q}\right\|_{W L^{1, \Phi}} \leq C\left\|\left(\sum_{j}\left|f_{j}\right|^{q}\right)^{1 / q}\right\|_{L^{1, \Phi}} .
$$

Theorem 22. Let $1<s<\infty$ and let $T_{\Omega}$ be a singular integral operator with kernel $\Omega \in\left(D_{s}\right)$. Assume that $\Phi$ satisfies (33) and $1 \leq D(\Phi)<2^{n}$; then, for $s^{\prime} \leq p<\infty$ and all $1<q<\infty$, there exists a constant $C>0$ independent of $\vec{f}=\left(f_{1}, f_{2}, \ldots\right)$ such that

$$
\left\|\left(\sum_{j}\left|T_{\Omega}\left(f_{j}\right)\right|^{q}\right)^{1 / q}\right\|_{L^{p, \Phi}} \leq C\left\|\left(\sum_{j}\left|f_{j}\right|^{q}\right)^{1 / q}\right\|_{L^{p, \Phi}} .
$$

Throughout this paper, the letter $C$ always denotes a positive constant independent of the main parameters involved, but it may be different from line to line. By $A \sim B$, we mean that there exists a constant $C>1$ such that $1 / C \leq A / B \leq C$. Moreover, we will denote the conjugate exponent of $p>1$ by $p^{\prime}=p /(p-1)$.

\section{Boundedness in the Weighted Morrey Spaces}

\subsection{Proofs of Theorems 12, 13, and 14}

Proof of Theorem 12. Let $\left(\sum_{j}\left|f_{j}\right|^{q}\right)^{1 / q} \in L^{p, \kappa}(w)$ with $1<$ $p, q<\infty$, and $0<\kappa<1$. Fix a ball $B=B\left(x_{0}, r_{B}\right) \subseteq \mathbb{R}^{n}$ and decompose $f_{j}=f_{j}^{0}+f_{j}^{\infty}$, where $f_{j}^{0}=f_{j} \cdot \chi_{2 B}$ and $\chi_{2 B}$ denotes the characteristic function of $2 B=B\left(x_{0}, 2 r_{B}\right)$, $j=1,2, \ldots$. Then, we write

$$
\begin{aligned}
& \frac{1}{w(B)^{\kappa / p}}\left(\int_{B}\left(\sum_{j}\left|M\left(f_{j}\right)(x)\right|^{q}\right)^{p / q} w(x) d x\right)^{1 / p} \\
& \leq \frac{1}{w(B)^{\kappa / p}}\left(\int_{B}\left(\sum_{j}\left|M\left(f_{j}^{0}\right)(x)\right|^{q}\right)^{p / q} w(x) d x\right)^{1 / p} \\
& \quad+\frac{1}{w(B)^{\kappa / p}}\left(\int_{B}\left(\sum_{j}\left|M\left(f_{j}^{\infty}\right)(x)\right|^{q}\right)^{p / q} w(x) d x\right)^{1 / p} \\
& =I_{1}+I_{2} .
\end{aligned}
$$

Using Theorem 3 and inequality (7), we get

$$
\begin{aligned}
I_{1} & \leq \frac{1}{w(B)^{\kappa / p}}\left\|\left(\sum_{j}\left|M\left(f_{j}^{0}\right)\right|^{q}\right)^{1 / q}\right\|_{L_{w}^{p}} \\
& \leq C \cdot \frac{1}{w(B)^{\kappa / p}}\left(\int_{2 B}\left(\sum_{j}\left|f_{j}(x)\right|^{q}\right)^{p / q} w(x) d x\right)^{1 / p} \\
& \leq C\left\|\left(\sum_{j}\left|f_{j}\right|^{q}\right)^{1 / q}\right\|_{L^{p, \kappa}(w)} \cdot \frac{w(2 B)^{\kappa / p}}{w(B)^{\kappa / p}} \\
& \leq C\left\|\left(\sum_{j}\left|f_{j}\right|^{q}\right)^{1 / q}\right\|_{L^{p, \kappa}(w)} \cdot
\end{aligned}
$$

Let us now turn to estimate the other term $I_{2}$. For any $x \in B$ and any fixed ball $\overline{\mathscr{B}}=\overline{\mathscr{B}}(x, \bar{r})$, by definition (1), one can write

$$
M\left(f_{j}^{\infty}\right)(x)=\sup _{\bar{r}>0} \frac{1}{|\overline{\mathscr{B}}(x, \bar{r})|} \int_{\overline{\mathscr{B}}(x, \bar{r}) \cap\left(2 B\left(x_{0}, r_{B}\right)\right)^{c} \neq \emptyset}\left|f_{j}(y)\right| d y .
$$

A simple geometric observation shows that when $x \in$ $B\left(x_{0}, r_{B}\right)$ and $y \in \overline{\mathscr{B}}(x, \bar{r}) \cap\left(2 B\left(x_{0}, r_{B}\right)\right)^{c}$, then we have $B\left(x_{0}, r_{B}\right) \subseteq 2 \overline{\mathscr{B}}(x, \bar{r})=\overline{\mathscr{B}}(x, 2 \bar{r})$. This fact together with the $A_{p}$ condition and Hölder's inequality implies that

$$
\begin{gathered}
\left(\sum_{j}\left|\frac{1}{|\overline{\mathscr{B}}(x, \bar{r})|} \int_{\overline{\mathscr{B}}(x, \bar{r}) \cap\left(2 B\left(x_{0}, r_{B}\right)\right)^{c} \neq \emptyset}\right| f_{j}(y)|d y|^{q}\right)^{1 / q} \\
\leq \frac{1}{|\overline{\mathscr{B}}(x, \bar{r})|} \int_{\overline{\mathscr{B}}(x, \bar{r}) \cap\left(2 B\left(x_{0}, r_{B}\right)\right)^{c} \neq \emptyset}\left(\sum_{j}\left|f_{j}(y)\right|^{q}\right)^{1 / q} d y \\
\leq \frac{1}{|\overline{\mathscr{B}}(x, \bar{r})|}\left(\int_{\overline{\mathscr{B}}(x, \bar{r})}\left(\sum_{j}\left|f_{j}(y)\right|^{q}\right)^{p / q} w(y) d y\right)^{1 / p}
\end{gathered}
$$




$$
\begin{aligned}
& \times\left(\int_{\overline{\mathscr{B}}(x, \bar{r})} w(y)^{-p^{\prime} / p} d y\right)^{1 / p^{\prime}} \\
\leq & C\left\|\left(\sum_{j}\left|f_{j}\right|^{q}\right)^{1 / q}\right\|_{L^{p, \kappa}(w)} w(\overline{\mathscr{B}}(x, \bar{r}))^{(\kappa-1) / p} \\
\leq & C\left\|\left(\sum_{j}\left|f_{j}\right|^{q}\right)^{1 / q}\right\|_{L^{p, \kappa}(w)} w\left(B\left(x_{0}, r_{B}\right)\right)^{(\kappa-1) / p} .
\end{aligned}
$$

Therefore, for any $x \in B$,

$$
\begin{aligned}
& \left(\sum_{j}\left|M\left(f_{j}^{\infty}\right)(x)\right|^{q}\right)^{1 / q} \\
& \leq C\left\|\left(\sum_{j}\left|f_{j}\right|^{q}\right)^{1 / q}\right\|_{L^{p, \kappa}(w)} w\left(B\left(x_{0}, r_{B}\right)\right)^{(\kappa-1) / p} .
\end{aligned}
$$

So we have

$$
\begin{aligned}
I_{2} & \leq\left(\sum_{j}\left|M\left(f_{j}^{\infty}\right)(x)\right|^{q}\right)^{1 / q} w\left(B\left(x_{0}, r_{B}\right)\right)^{(1-\kappa) / p} \\
& \leq C\left\|\left(\sum_{j}\left|f_{j}\right|^{q}\right)^{1 / q}\right\|_{L^{p, \kappa}(w)} .
\end{aligned}
$$

Combining the above estimates for $I_{1}$ and $I_{2}$ and then taking the supremum over all balls $B \subseteq \mathbb{R}^{n}$, we complete the proof of Theorem 12.

Proof of Theorem 13. Let $\left(\sum_{j}\left|f_{j}\right|^{q}\right)^{1 / q} \in L^{1, \kappa}(w)$ with $1<q<$ $\infty$ and $0<\kappa<1$. Fix a ball $B=B\left(x_{0}, r_{B}\right) \subseteq \mathbb{R}^{n}$; then, we set $f_{j}=f_{j}^{0}+f_{j}^{\infty}$, where $f_{j}^{0}=f_{j} \cdot \chi_{2 B}, j=1,2, \ldots$. Then, for any given $\lambda>0$, one writes

$$
\begin{aligned}
& w\left(\left\{x \in B:\left(\sum_{j}\left|M\left(f_{j}\right)(x)\right|^{q}\right)^{1 / q}>\lambda\right\}\right) \\
& \leq w\left(\left\{x \in B:\left(\sum_{j}\left|M\left(f_{j}^{0}\right)(x)\right|^{q}\right)^{1 / q}>\frac{\lambda}{2}\right\}\right) \\
& +w\left(\left\{x \in B:\left(\sum_{j}\left|M\left(f_{j}^{\infty}\right)(x)\right|^{q}\right)^{1 / q}>\frac{\lambda}{2}\right\}\right) \\
& =I_{1}^{\prime}+I_{2}^{\prime} .
\end{aligned}
$$

Theorem 4 and inequality (7) yield

$$
\begin{aligned}
I_{1}^{\prime} & \leq \frac{2}{\lambda} \cdot\left\|\left(\sum_{j}\left|M\left(f_{j}^{0}\right)\right|^{q}\right)^{1 / q}\right\|_{W L_{w}^{1}} \\
& \leq \frac{C}{\lambda} \cdot\left(\int_{2 B}\left(\sum_{j}\left|f_{j}(x)\right|^{q}\right)^{1 / q} w(x) d x\right) \\
& \leq \frac{C \cdot w(2 B)^{\kappa}}{\lambda}\left\|\left(\sum_{j}\left|f_{j}\right|^{q}\right)^{1 / q}\right\|_{L^{1, \kappa}(w)} \\
& \leq \frac{C \cdot w(B)^{\kappa}}{\lambda}\left\|\left(\sum_{j}\left|f_{j}\right|^{q}\right)^{1 / q}\right\|_{L^{1, \kappa}(w)} .
\end{aligned}
$$

Next, let us turn to deal with the term $I_{2}^{\prime}$. We apply Chebyshev's inequality to obtain

$$
\begin{aligned}
I_{2}^{\prime}= & \int_{\left\{x \in B:\left(\sum_{j}\left|M\left(f_{j}^{\infty}\right)(x)\right|^{q}\right)^{1 / q}>\lambda / 2\right\}} w(x) d x \\
\leq & \frac{2}{\lambda} \\
& \times \int_{\left\{x \in B:\left(\sum_{j}\left|M\left(f_{j}^{\infty}\right)(x)\right|^{q}\right)^{1 / q}>\lambda / 2\right\}}\left(\sum_{j}\left|M\left(f_{j}^{\infty}\right)(x)\right|^{q}\right)^{1 / q} \\
& \quad \times w(x) d x .
\end{aligned}
$$

For any $x \in B$ and any fixed ball $\overline{\mathscr{B}}=\overline{\mathscr{B}}(x, \bar{r})$, we still have $B\left(x_{0}, r_{B}\right) \subseteq 2 \overline{\mathscr{B}}(x, \bar{r})=\overline{\mathscr{B}}(x, 2 \bar{r})$ if $x \in B\left(x_{0}, r_{B}\right)$ and $y \in$ $\overline{\mathscr{B}}(x, \bar{r}) \cap\left(2 B\left(x_{0}, r_{B}\right)\right)^{c}$. It then follows from (49) and the $A_{1}$ condition that

$$
\begin{aligned}
& \left(\sum_{j}\left|\frac{1}{|\overline{\mathscr{B}}(x, \bar{r})|} \int_{\overline{\mathscr{B}}(x, \bar{r}) \cap\left(2 B\left(x_{0}, r_{B}\right)\right)^{c} \neq \emptyset}\right| f_{j}(y)|d y|^{q}\right)^{1 / q} \\
& \quad \leq \frac{1}{|\overline{\mathscr{B}}(x, \bar{r})|} \int_{\overline{\mathscr{B}}(x, \bar{r})}\left(\sum_{j}\left|f_{j}(y)\right|^{q}\right)^{1 / q} d y \\
& \leq \frac{\operatorname{essinf}_{y \in \overline{\mathscr{B}}(x, \bar{r})} w(y)}{w(\overline{\mathscr{B}}(x, \bar{r}))} \int_{\overline{\mathscr{B}}(x, \bar{r})}\left(\sum_{j}\left|f_{j}(y)\right|^{q}\right)^{1 / q} d y \\
& \leq \frac{1}{w(\overline{\mathscr{B}}(x, \bar{r}))} \int_{\overline{\mathscr{B}}(x, \bar{r})}\left(\sum_{j}\left|f_{j}(y)\right|^{q}\right)^{1 / q} w(y) d y \\
& \quad \leq\left\|\left(\sum_{j}\left|f_{j}\right|^{q}\right)^{1 / q}\right\|_{L^{1, \kappa}(w)} w(\overline{\mathscr{B}}(x, \bar{r}))^{(\kappa-1)} \\
& \leq\left\|\left(\sum_{j}\left|f_{j}\right|^{q}\right)^{1 / q}\right\|_{L^{1, \kappa}(w)} w\left(B\left(x_{0}, r_{B}\right)\right)^{(\kappa-1)} .
\end{aligned}
$$


Hence, for any $x \in B$,

$$
\begin{aligned}
& \left(\sum_{j}\left|M\left(f_{j}^{\infty}\right)(x)\right|^{q}\right)^{1 / q} \\
& \leq\left\|\left(\sum_{j}\left|f_{j}\right|^{q}\right)^{1 / q}\right\|_{L^{1, \kappa}(w)} w\left(B\left(x_{0}, r_{B}\right)\right)^{(\kappa-1)} .
\end{aligned}
$$

Therefore,

$$
\begin{aligned}
I_{2}^{\prime} & \leq C\left\|\left(\sum_{j}\left|f_{j}\right|^{q}\right)^{1 / q}\right\|_{L^{1, \kappa}(w)} w(B)^{(\kappa-1)} \cdot \frac{2}{\lambda} \int_{B} w(x) d x \\
& \leq \frac{C \cdot w(B)^{\kappa}}{\lambda}\left\|\left(\sum_{j}\left|f_{j}\right|^{q}\right)^{1 / q}\right\|_{L^{1, \kappa}(w)} .
\end{aligned}
$$

Summing up the above estimates for $I_{1}^{\prime}$ and $I_{2}^{\prime}$ and then taking the supremum over all balls $B \subseteq \mathbb{R}^{n}$ and all $\lambda>0$, we finish the proof of Theorem 13 .

Proof of Theorem 14. Let $\left(\sum_{j}\left|f_{j}\right|^{q}\right)^{1 / q} \in L^{p, \kappa}(w)$ with $1<$ $p, q<\infty$, and $0<\kappa<1$. For any ball $B=B\left(x_{0}, r_{B}\right) \subseteq \mathbb{R}^{n}$ and let $f_{j}=f_{j}^{0}+f_{j}^{\infty}$, where $f_{j}^{0}=f_{j} \cdot \chi_{2 B}, j=1,2, \ldots$. Then, we have

$$
\begin{aligned}
& \frac{1}{w(B)^{\kappa / p}}\left(\int_{B}\left(\sum_{j}\left|M_{w}\left(f_{j}\right)(x)\right|^{q}\right)^{p / q} w(x) d x\right)^{1 / p} \\
& \leq \frac{1}{w(B)^{\kappa / p}}\left(\int_{B}\left(\sum_{j}\left|M_{w}\left(f_{j}^{0}\right)(x)\right|^{q}\right)^{p / q} w(x) d x\right)^{1 / p} \\
& \quad+\frac{1}{w(B)^{\kappa / p}}\left(\int_{B}\left(\sum_{j}\left|M_{w}\left(f_{j}^{\infty}\right)(x)\right|^{q}\right)^{p / q} w(x) d x\right)^{1 / p} \\
& =I_{1}^{\prime \prime}+I_{2}^{\prime \prime} .
\end{aligned}
$$

By using Theorem $5^{\prime}$ and inequality (7), we thus obtain

$$
\begin{aligned}
I_{1}^{\prime \prime} & \leq \frac{1}{w(B)^{\kappa / p}}\left\|\left(\sum_{j}\left|M_{w}\left(f_{j}^{0}\right)\right|^{q}\right)^{1 / q}\right\|_{L_{w}^{p}} \\
& \leq C \cdot \frac{1}{w(B)^{\kappa / p}}\left(\int_{2 B}\left(\sum_{j}\left|f_{j}(x)\right|^{q}\right)^{p / q} w(x) d x\right)^{1 / p}
\end{aligned}
$$

$$
\begin{aligned}
& \leq C\left\|\left(\sum_{j}\left|f_{j}\right|^{q}\right)^{1 / q}\right\|_{L^{p, \kappa}(w)} \cdot \frac{w(2 B)^{\kappa / p}}{w(B)^{\kappa / p}} \\
& \leq C\left\|\left(\sum_{j}\left|f_{j}\right|^{q}\right)^{1 / q}\right\|_{L^{p, \kappa}(w)} \cdot
\end{aligned}
$$

To estimate the other term $I_{2}^{\prime \prime}$, it follows from definition (13) that, for any $x \in B$ and any fixed ball $\overline{\mathscr{B}}=\overline{\mathscr{B}}(x, \bar{r})$,

$$
\begin{aligned}
& M_{w}\left(f_{j}^{\infty}\right)(x) \\
& \quad=\sup _{\bar{r}>0} \frac{1}{w(\overline{\mathscr{B}}(x, \bar{r}))} \int_{\overline{\mathscr{B}}(x, \bar{r}) \cap\left(2 B\left(x_{0}, r_{B}\right)\right)^{c} \neq \emptyset}\left|f_{j}(y)\right| w(y) d y .
\end{aligned}
$$

Moreover, as before, we know that $B\left(x_{0}, r_{B}\right) \subseteq 2 \overline{\mathscr{B}}(x, \bar{r})=$ $\overline{\mathscr{B}}(x, 2 \bar{r})$ if $x \in B\left(x_{0}, r_{B}\right)$ and $y \in \overline{\mathscr{B}}(x, \bar{r}) \cap\left(2 B\left(x_{0}, r_{B}\right)\right)^{c}$. Applying the $\Delta_{2}$ condition and Hölder's inequality, we can deduce that

$$
\begin{aligned}
& \left(\sum_{j}\left|\frac{1}{w(\overline{\mathscr{B}}(x, \bar{r}))} \int_{\overline{\mathscr{B}}(x, \bar{r}) \cap\left(2 B\left(x_{0}, r_{B}\right)\right)^{c} \neq \emptyset}\right| f_{j}(y)|w(y) d y|^{q}\right)^{1 / q} \\
& \leq \frac{1}{w(\overline{\mathscr{B}}(x, \bar{r}))} \int_{\overline{\mathscr{B}}(x, \bar{r})}\left(\sum_{j}\left|f_{j}(y)\right|^{q}\right)^{1 / q} w(y) d y \\
& \leq \frac{1}{w(\overline{\mathscr{B}}(x, \bar{r}))}\left(\int_{\overline{\mathscr{B}}(x, \bar{r})}\left(\sum_{j}\left|f_{j}(y)\right|^{q}\right)^{p / q} w(y) d y\right)^{1 / p} \\
& \quad \times\left(\int_{\overline{\mathscr{B}}(x, \bar{r})} w(y) d y\right)^{1 / p^{\prime}} \\
& \leq \\
& \quad\left\|\left(\sum_{j}\left|f_{j}\right|^{q}\right)^{1 / q}\right\|_{L^{p, \kappa}(w)} w(\overline{\mathscr{B}}(x, \bar{r}))^{(\kappa-1) / p} \\
& \leq\left\|\left(\sum_{j}\left|f_{j}\right|^{q}\right)^{1 / q}\right\|_{L^{p, \kappa}(w)} w\left(B\left(x_{0}, r_{B}\right)\right)^{(\kappa-1) / p} .
\end{aligned}
$$

Therefore, for any $x \in B$,

$$
\begin{aligned}
& \left(\sum_{j}\left|M_{w}\left(f_{j}^{\infty}\right)(x)\right|^{q}\right)^{1 / q} \\
& \leq C\left\|\left(\sum_{j}\left|f_{j}\right|^{q}\right)^{1 / q}\right\|_{L^{p, \kappa}(w)} w\left(B\left(x_{0}, r_{B}\right)\right)^{(\kappa-1) / p} .
\end{aligned}
$$


Consequently,

$$
\begin{aligned}
I_{2}^{\prime \prime} & \leq\left(\sum_{j}\left|M_{w}\left(f_{j}^{\infty}\right)(x)\right|^{q}\right)^{1 / q} w\left(B\left(x_{0}, r_{B}\right)\right)^{(1-\kappa) / p} \\
& \leq C\left\|\left(\sum_{j}\left|f_{j}\right|^{q}\right)^{1 / q}\right\|_{L^{p, \kappa}(w)} \cdot
\end{aligned}
$$

Collecting all the above estimates for $I_{1}^{\prime \prime}$ and $I_{2}^{\prime \prime}$ and then taking the supremum over all balls $B \subseteq \mathbb{R}^{n}$, we complete the proof of Theorem 14 .

\subsection{Proofs of Theorems 15,16 , and 17}

Proof of Theorem 15. Let $\left(\sum_{j}\left|f_{j}\right|^{q}\right)^{1 / q} \in L^{p, \kappa}(w)$ with $1<$ $p, q<\infty$, and $0<\kappa<1$. Fix a ball $B=B\left(x_{0}, r_{B}\right) \subseteq \mathbb{R}^{n}$ and decompose $f_{j}=f_{j}^{0}+f_{j}^{\infty}$, where $f_{j}^{0}=f_{j} \cdot \chi_{2 B}, j=1,2, \ldots$. Then, we write

$$
\begin{gathered}
\frac{1}{w(B)^{\kappa / p}}\left(\int_{B}\left(\sum_{j}\left|T_{\Omega}\left(f_{j}\right)(x)\right|^{q}\right)^{p / q} w(x) d x\right)^{1 / p} \\
\leq \frac{1}{w(B)^{\kappa / p}}\left(\int_{B}\left(\sum_{j}\left|T_{\Omega}\left(f_{j}^{0}\right)(x)\right|^{q}\right)^{p / q} w(x) d x\right)^{1 / p} \\
+\frac{1}{w(B)^{\kappa / p}}\left(\int_{B}\left(\sum_{j}\left|T_{\Omega}\left(f_{j}^{\infty}\right)(x)\right|^{q}\right)^{p / q} w(x) d x\right)^{1 / p} \\
=J_{1}+J_{2} .
\end{gathered}
$$

It follows from Theorem 6 and inequality (7) that

$$
\begin{aligned}
J_{1} & \leq \frac{1}{w(B)^{\kappa / p}}\left\|\left(\sum_{j}\left|T_{\Omega}\left(f_{j}^{0}\right)\right|^{q}\right)^{1 / q}\right\|_{L_{w}^{p}} \\
& \leq C \cdot \frac{1}{w(B)^{\kappa / p}}\left(\int_{2 B}\left(\sum_{j}\left|f_{j}(x)\right|^{q}\right)^{p / q} w(x) d x\right)^{1 / p} \\
& \leq C\left\|\left(\sum_{j}\left|f_{j}\right|^{q}\right)^{1 / q}\right\|_{L^{p, \kappa}(w)} \cdot \frac{w(2 B)^{\kappa / p}}{w(B)^{\kappa / p}} \\
& \leq C\left\|\left(\sum_{j}\left|f_{j}\right|^{q}\right)^{1 / q}\right\|_{L^{p, \kappa}(w)} .
\end{aligned}
$$

To estimate the term $J_{2}$, we first observe that when $x \in B$ and $y \in(2 B)^{c}$, then $|y-x| \sim\left|y-x_{0}\right|$. This fact together with the condition $\Omega \in L^{\infty}\left(S^{n-1}\right)$ yields

$$
\begin{aligned}
& \left|T_{\Omega}\left(f_{j}^{\infty}\right)(x)\right| \\
& \quad=\left|\int_{(2 B)^{c}} \frac{\Omega(x-y)}{|x-y|^{n}} f_{j}(y) d y\right| \\
& \quad \leq C \sum_{\ell=1}^{\infty} \int_{2^{\ell+1} B \backslash 2^{\ell} B} \frac{1}{|x-y|^{n}} \cdot\left|f_{j}(y)\right| d y \\
& \quad \leq C \sum_{\ell=1}^{\infty} \frac{1}{\left|2^{\ell+1} B\right|} \int_{2^{\ell+1} B \backslash 2^{\ell} B}\left|f_{j}(y)\right| d y .
\end{aligned}
$$

Then, by duality and Hölder's inequality with exponent $q>1$, we get

$$
\begin{aligned}
& \left(\sum_{j}\left|T_{\Omega}\left(f_{j}^{\infty}\right)(x)\right|^{q}\right)^{1 / q} \\
& \leq C\left(\sum_{j}\left|\sum_{\ell=1}^{\infty} \frac{1}{\left|2^{\ell+1} B\right|} \int_{2^{\ell+1} B \backslash 2^{\ell} B}\right| f_{j}(y)|d y|^{q}\right)^{1 / q} \\
& \leq C \sup _{\left(\sum_{j}\left|\zeta_{j}\right|^{\prime}\right)^{1 / q^{\prime}} \leq 1} \sum_{j}\left(\sum_{\ell=1}^{\infty} \frac{1}{\left|2^{\ell+1} B\right|} \int_{2^{\ell+1} B}\left|f_{j}(y)\right| d y \cdot\left|\zeta_{j}\right|\right) \\
& \leq C \sum_{\ell=1}^{\infty} \frac{1}{\left|2^{\ell+1} B\right|} \int_{2^{\ell+1} B}\left(\sum_{j} \mid \zeta_{j} q^{q^{\prime}}\right)^{1 / q^{\prime}} \leq 1 \\
& \leq C \sum_{\ell=1}^{\infty} \frac{1}{\left|2^{\ell+1} B\right|} \int_{2^{\ell+1} B}\left(\sum_{j}\left|f_{j}(y)\right|^{q}\right)^{1 / q} d y .
\end{aligned}
$$

Furthermore, it follows from Hölder's inequality, (68), and the $A_{p}$ condition that

$$
\begin{aligned}
& \left(\sum_{j}\left|T_{\Omega}\left(f_{j}^{\infty}\right)(x)\right|^{q}\right)^{1 / q} \\
& \leq C \sum_{\ell=1}^{\infty} \frac{1}{\left|2^{\ell+1} B\right|}\left(\int_{2^{\ell+1} B}\left(\sum_{j}\left|f_{j}(y)\right|^{q}\right)^{p / q} w(y) d y\right)^{1 / p} \\
& \quad \times\left(\int_{2^{\ell+1} B} w(y)^{-p^{\prime} / p} d y\right)^{1 / p^{\prime}} \\
& \leq C\left\|\left(\sum_{j}\left|f_{j}\right|^{q}\right)^{1 / q}\right\|_{L^{p, \kappa}(w)} \cdot \sum_{\ell=1}^{\infty} w\left(2^{\ell+1} B\right)^{(\kappa-1) / p} .
\end{aligned}
$$


Since $w \in A_{p} \subset A_{\infty}$, thus, by inequality (8), we find that

$$
\begin{aligned}
J_{2} & \leq C\left\|\left(\sum_{j}\left|f_{j}\right|^{q}\right)^{1 / q}\right\|_{L^{p, \kappa}(w)} \cdot \sum_{\ell=1}^{\infty} \frac{w(B)^{(1-\kappa) / p}}{w\left(2^{\ell+1} B\right)^{(1-\kappa) / p}} \\
& \leq C\left\|\left(\sum_{j}\left|f_{j}\right|^{q}\right)^{1 / q}\right\|_{L^{p, \kappa}(w)} \cdot \sum_{\ell=1}^{\infty}\left(\frac{|B|}{\left|2^{\ell+1} B\right|}\right)^{\delta \cdot(1-\kappa) / p} \\
& \leq C\left\|\left(\sum_{j}\left|f_{j}\right|^{q}\right)^{1 / q}\right\|_{L^{p, \kappa}(w)},
\end{aligned}
$$

where the last series is convergent since $0<\kappa<1$ and $\delta>0$. Combining the above estimates for $J_{1}$ and $J_{2}$ and then taking the supremum over all balls $B \subseteq \mathbb{R}^{n}$, we complete the proof of Theorem 15.

Proof of Theorem 16. Let $\left(\sum_{j}\left|f_{j}\right|^{q}\right)^{1 / q} \in L^{1, \kappa}(w)$ with $1<q<$ $\infty$ and $0<\kappa<1$. For any ball $B=B\left(x_{0}, r_{B}\right) \subseteq \mathbb{R}^{n}$ and decompose $f_{j}=f_{j}^{0}+f_{j}^{\infty}$, where $f_{j}^{0}=f_{j} \cdot \chi_{2 B}, j=1,2, \ldots$. Then, for any given $\lambda>0$, we can write

$$
\begin{aligned}
& w\left(\left\{x \in B:\left(\sum_{j}\left|T_{\Omega}\left(f_{j}\right)(x)\right|^{q}\right)^{1 / q}>\lambda\right\}\right) \\
& \leq w\left(\left\{x \in B:\left(\sum_{j}\left|T_{\Omega}\left(f_{j}^{0}\right)(x)\right|^{q}\right)^{1 / q}>\frac{\lambda}{2}\right\}\right) \\
& +w\left(\left\{x \in B:\left(\sum_{j}\left|T_{\Omega}\left(f_{j}^{\infty}\right)(x)\right|^{q}\right)^{1 / q}>\frac{\lambda}{2}\right\}\right) \\
& =J_{1}^{\prime}+J_{2}^{\prime} .
\end{aligned}
$$

Theorem 7 and inequality (7) imply that

$$
\begin{aligned}
J_{1}^{\prime} & \leq \frac{2}{\lambda} \cdot\left\|\left(\sum_{j}\left|T_{\Omega}\left(f_{j}^{0}\right)\right|^{q}\right)^{1 / q}\right\|_{W L_{w}^{1}} \\
& \leq \frac{C}{\lambda} \cdot\left(\int_{2 B}\left(\sum_{j}\left|f_{j}(x)\right|^{q}\right)^{1 / q} w(x) d x\right) \\
& \leq \frac{C \cdot w(2 B)^{\kappa}}{\lambda}\left\|\left(\sum_{j}\left|f_{j}\right|^{q}\right)^{1 / q}\right\|_{L^{1, \kappa}(w)} \\
& \leq \frac{C \cdot w(B)^{\kappa}}{\lambda}\left\|\left(\sum_{j}\left|f_{j}\right|^{q}\right)^{1 / q}\right\|_{L^{1, \kappa}(w)} .
\end{aligned}
$$

In the proof of Theorem 15, we have already showed that, for any $x \in B$,

$$
\begin{aligned}
& \left(\sum_{j}\left|T_{\Omega}\left(f_{j}^{\infty}\right)(x)\right|^{q}\right)^{1 / q} \\
& \leq C \sum_{\ell=1}^{\infty} \frac{1}{\left|2^{\ell+1} B\right|} \int_{2^{\ell+1} B}\left(\sum_{j}\left|f_{j}(y)\right|^{q}\right)^{1 / q} d y .
\end{aligned}
$$

On the other hand, it follows directly from the $A_{1}$ condition that

$$
\begin{aligned}
& \left(\sum_{j}\left|T_{\Omega}\left(f_{j}^{\infty}\right)(x)\right|^{q}\right)^{1 / q} \\
& \leq C \sum_{\ell=1}^{\infty} \frac{\operatorname{essinf}_{y \in 2^{\ell+1} B} w(y)}{w\left(2^{\ell+1} B\right)} \int_{2^{\ell+1} B}\left(\sum_{j}\left|f_{j}(y)\right|^{q}\right)^{1 / q} d y \\
& \leq C \sum_{\ell=1}^{\infty} \frac{1}{w\left(2^{\ell+1} B\right)} \int_{2^{\ell+1} B}\left(\sum_{j}\left|f_{j}(y)\right|^{q}\right)^{1 / q} w(y) d y \\
& \leq C\left\|\left(\sum_{j}\left|f_{j}\right|^{q}\right)^{1 / q}\right\|_{L^{1, \kappa}(w)} \sum_{\ell=1}^{\infty} \frac{1}{w\left(2^{\ell+1} B\right)^{1-\kappa}} .
\end{aligned}
$$

In addition, since $w \in A_{1} \subset A_{\infty}$, then by inequality (8), we can see that for all $x \in B$

$$
\begin{aligned}
& \left(\sum_{j}\left|T_{\Omega}\left(f_{j}^{\infty}\right)(x)\right|^{q}\right)^{1 / q} \\
& \leq C\left\|\left(\sum_{j}\left|f_{j}\right|^{q}\right)^{1 / q}\right\|_{L^{1, \kappa}(w)} \cdot \frac{1}{w(B)^{1-\kappa}} \sum_{\ell=1}^{\infty} \frac{w(B)^{1-\kappa}}{w\left(2^{\ell+1} B\right)^{1-\kappa}} \\
& \leq C\left\|\left(\sum_{j}\left|f_{j}\right|^{q}\right)^{1 / q}\right\|_{L^{1, \kappa}(w)} \cdot \frac{1}{w(B)^{1-\kappa}} \sum_{\ell=1}^{\infty}\left(\frac{|B|}{\left|2^{\ell+1} B\right|}\right)^{\delta^{*} \cdot(1-\kappa)} \\
& \leq C\left\|\left(\sum_{j}\left|f_{j}\right|^{q}\right)^{1 / q}\right\|_{L^{1, \kappa}(w)} \cdot \frac{1}{w(B)^{1-\kappa}},
\end{aligned}
$$

where in the last inequality we have used the fact that $\delta^{*} \cdot(1-$ $\kappa)>0$. If

$$
\left\{x \in B:\left(\sum_{j}\left|T_{\Omega}\left(f_{j}^{\infty}\right)(x)\right|^{q}\right)^{1 / q}>\frac{\lambda}{2}\right\}=\emptyset,
$$

then the inequality

$$
J_{2}^{\prime} \leq \frac{C \cdot w(B)^{\kappa}}{\lambda}\left\|\left(\sum_{j}\left|f_{j}\right|^{q}\right)^{1 / q}\right\|_{L^{1, \kappa}(w)}
$$


10

International Journal of Mathematics and Mathematical Sciences

holds trivially. Now, we may suppose that

$$
\left\{x \in B:\left(\sum_{j}\left|T_{\Omega}\left(f_{j}^{\infty}\right)(x)\right|^{q}\right)^{1 / q}>\frac{\lambda}{2}\right\} \neq \emptyset .
$$

Then, by the pointwise inequality (75), we can see that

$$
\lambda \leq C\left\|\left(\sum_{j}\left|f_{j}\right|^{q}\right)^{1 / q}\right\|_{L^{1, \kappa}(w)} \cdot \frac{1}{w(B)^{1-\kappa}}
$$

which in turn is equivalent to

$$
w(B) \leq \frac{C \cdot w(B)^{\kappa}}{\lambda}\left\|\left(\sum_{j}\left|f_{j}\right|^{q}\right)^{1 / q}\right\|_{L^{1, \kappa}(w)} .
$$

Therefore,

$$
J_{2}^{\prime} \leq w(B) \leq \frac{C \cdot w(B)^{\kappa}}{\lambda}\left\|\left(\sum_{j}\left|f_{j}\right|^{q}\right)^{1 / q}\right\|_{L^{1, \kappa}(w)} .
$$

Summing up the above estimates for $J_{1}^{\prime}$ and $J_{2}^{\prime}$ and then taking the supremum over all balls $B=B\left(x_{0}, r_{B}\right) \subseteq \mathbb{R}^{n}$ and all $\lambda>0$, we conclude the proof of Theorem 16.

Proof of Theorem 17. Let $\left(\sum_{j}\left|f_{j}\right|^{q}\right)^{1 / q} \in L^{p, \kappa}(w)$ with $1<$ $p, q<\infty$, and $0<\kappa<1$. For each fixed ball $B=B\left(x_{0}, r_{B}\right) \subseteq$ $\mathbb{R}^{n}$, we set $f_{j}=f_{j}^{0}+f_{j}^{\infty}$, where $f_{j}^{0}=f_{j} \cdot \chi_{2 B}, j=1,2, \ldots$. Then, we have

$$
\begin{aligned}
& \frac{1}{w(B)^{\kappa / p}}\left(\int_{B}\left(\sum_{j}\left|T_{\Omega}\left(f_{j}\right)(x)\right|^{q}\right)^{p / q} w(x) d x\right)^{1 / p} \\
& \leq \frac{1}{w(B)^{\kappa / p}}\left(\int_{B}\left(\sum_{j}\left|T_{\Omega}\left(f_{j}^{0}\right)(x)\right|^{q}\right)^{p / q} w(x) d x\right)^{1 / p} \\
& \quad+\frac{1}{w(B)^{\kappa / p}}\left(\int_{B}\left(\sum_{j}\left|T_{\Omega}\left(f_{j}^{\infty}\right)(x)\right|^{q}\right)^{p / q} w(x) d x\right)^{1 / p} \\
& =J_{1}^{\prime \prime}+J_{2}^{\prime \prime} .
\end{aligned}
$$

Applying Theorem 8 and inequality (7), we can get

$$
\begin{aligned}
J_{1}^{\prime \prime} & \leq \frac{1}{w(B)^{\kappa / p}}\left\|\left(\sum_{j}\left|T_{\Omega}\left(f_{j}^{0}\right)\right|^{q}\right)^{1 / q}\right\|_{L_{w}^{p}} \\
& \leq C \cdot \frac{1}{w(B)^{\kappa / p}}\left(\int_{2 B}\left(\sum_{j}\left|f_{j}(x)\right|^{q}\right)^{p / q} w(x) d x\right)^{1 / p}
\end{aligned}
$$

$$
\begin{aligned}
& \leq C\left\|\left(\sum_{j}\left|f_{j}\right|^{q}\right)^{1 / q}\right\|_{L^{p, \kappa}(w)} \cdot \frac{w(2 B)^{\kappa / p}}{w(B)^{\kappa / p}} \\
& \leq C\left\|\left(\sum_{j}\left|f_{j}\right|^{q}\right)^{1 / q}\right\|_{L^{p, \kappa}(w)} \cdot
\end{aligned}
$$

We now turn to deal with the term $J_{2}^{\prime \prime}$. An application of Hölder's inequality gives us that

$$
\begin{aligned}
& \left(\sum_{j}\left|T_{\Omega}\left(f_{j}^{\infty}\right)(x)\right|^{q}\right)^{1 / q} \\
& \leq\left|\int_{(2 B)^{c}} \frac{\Omega(x-y)}{|x-y|^{n}}\left(\sum_{j}\left|f_{j}(y)\right|^{q}\right)^{1 / q} d y\right| \\
& \leq \sum_{\ell=1}^{\infty}\left(\int_{2^{\ell+1} B \backslash 2^{\ell} B}|\Omega(x-y)|^{s} d y\right)^{1 / s} \\
& \quad \times\left(\int_{2^{\ell+1} B \backslash 2^{\ell} B} \frac{1}{|x-y|^{n s^{\prime}}}\left(\sum_{j}\left|f_{j}(y)\right|^{q}\right)^{s^{\prime} / q} d y\right)^{1 / s^{\prime}} .
\end{aligned}
$$

When $x \in B$ and $y \in 2^{\ell+1} B \backslash 2^{\ell} B$, then we can easily see that $2^{\ell-1} r_{B} \leq|y-x|<2^{\ell+2} r_{B}$. Since $\Omega \in L^{s}\left(S^{n-1}\right)$ with $1<s<\infty$, then

$$
\begin{aligned}
& \left(\int_{2^{\ell+1} B \backslash 2^{\ell} B}|\Omega(x-y)|^{s} d y\right)^{1 / s} \\
& \quad \leq\left(\int_{2^{\ell-1} r_{B}}^{\infty} \int_{S^{n-1}}\left|\Omega\left(z^{\prime}\right)\right|^{s} d \sigma\left(z^{\prime}\right) \rho^{n-1} d \rho\right)^{1 / s} \\
& \quad \leq C\|\Omega\|_{L^{s}\left(S^{n-1}\right)}\left|2^{\ell+1} B\right|^{1 / s} .
\end{aligned}
$$

Consequently,

$$
\begin{aligned}
& \left(\sum_{j}\left|T_{\Omega}\left(f_{j}^{\infty}\right)(x)\right|^{q}\right)^{1 / q} \\
& \leq C\|\Omega\|_{L^{s}\left(S^{n-1}\right)} \sum_{\ell=1}^{\infty}\left|2^{\ell+1} B\right|^{1 / s} \cdot \frac{1}{\left|2^{\ell+1} B\right|} \\
& \quad \times\left(\int_{2^{\ell+1} B}\left(\sum_{j}\left|f_{j}(y)\right|^{q}\right)^{s^{\prime} / q} d y\right)^{1 / s^{\prime}} \\
& =C\|\Omega\|_{L^{s}\left(S^{n-1}\right)} \\
& \quad \times \sum_{\ell=1}^{\infty}\left(\frac{1}{\left|2^{\ell+1} B\right|} \int_{2^{\ell+1} B}\left(\sum_{j}\left|f_{j}(y)\right|^{q}\right)^{s^{\prime} / q} d y\right)^{1 / s^{\prime}} .
\end{aligned}
$$


We will consider two cases. When $p=s^{\prime}$, then it follows directly from the $A_{1}$ condition that

$$
\begin{aligned}
& \left(\sum_{j}\left|T_{\Omega}\left(f_{j}^{\infty}\right)(x)\right|^{q}\right)^{1 / q} \\
& \leq C \sum_{\ell=1}^{\infty}\left(\frac{1}{w\left(2^{\ell+1} B\right)} \int_{2^{\ell+1} B}\left(\sum_{j}\left|f_{j}(y)\right|^{q}\right)^{p / q} w(y) d y\right)^{1 / p} .
\end{aligned}
$$

For the case of $p>s^{\prime}$, we set $t=p / s^{\prime}>1$. Then, by using Hölder's inequality with exponent $t>1$ and the fact that $w \in$ $A_{t} \subset A_{\infty}$, we deduce that

$$
\begin{gathered}
\left(\sum_{j}\left|T_{\Omega}\left(f_{j}^{\infty}\right)(x)\right|^{q}\right)^{1 / q} \\
\leq C \sum_{\ell=1}^{\infty} \frac{1}{\left|2^{\ell+1} B\right|^{1 / s^{\prime}}}\left(\int_{2^{\ell+1} B}\left(\sum_{j}\left|f_{j}(y)\right|^{q}\right)^{p / q} w(y) d y\right)^{1 / p} \\
\quad \times\left(\int_{2^{\ell+1} B} w(y)^{-t^{\prime} / t} d y\right)^{1 / t^{\prime} s^{\prime}} \\
\leq C \sum_{\ell=1}^{\infty}\left(\frac{1}{w\left(2^{\ell+1} B\right)} \int_{2^{\ell+1} B}\left(\sum_{j}\left|f_{j}(y)\right|^{q}\right)^{p / q} w(y) d y\right)^{1 / p} .
\end{gathered}
$$

Hence, for every $s^{\prime} \leq p<\infty$, by the pointwise estimates (87) and (88) together with inequality (8), we finally obtain

$$
\begin{aligned}
J_{2}^{\prime \prime} \leq & C \sum_{\ell=1}^{\infty}\left(\frac{1}{w\left(2^{\ell+1} B\right)} \int_{2^{\ell+1} B}\left(\sum_{j}\left|f_{j}(y)\right|^{q}\right)^{p / q} w(y) d y\right)^{1 / p} \\
& \cdot w(B)^{(1-\kappa) / p} \\
\leq & C\left\|\left(\sum_{j}\left|f_{j}\right|^{q}\right)^{1 / q}\right\|_{L^{p, \kappa}(w)} \cdot \sum_{\ell=1}^{\infty} \frac{w(B)^{(1-\kappa) / p}}{w\left(2^{\ell+1} B\right)^{(1-\kappa) / p}} \\
\leq & C\left\|\left(\sum_{j}\left|f_{j}\right|^{q}\right)^{1 / q}\right\|_{L^{p, \kappa}(w)} \cdot \sum_{\ell=1}^{\infty}\left(\frac{|B|}{\left|2^{\ell+1} B\right|}\right)^{\delta^{* *} \cdot(1-\kappa) / p} \\
\leq & C\left\|\left(\sum_{j}\left|f_{j}\right|^{q}\right)^{1 / q}\right\|_{L^{p, \kappa}(w)} \cdot
\end{aligned}
$$

Combining the above estimates for $J_{1}^{\prime \prime}$ and $J_{2}^{\prime \prime}$ and then taking the supremum over all balls $B \subseteq \mathbb{R}^{n}$, we are done.

\section{Boundedness in the Generalized Morrey Spaces}

4.1. Proofs of Theorems 18 and 19. In order to prove the main theorems of this section, we need to establish the following technical lemma.

Lemma 23. Let $\left(\sum_{j}\left|f_{j}\right|^{q}\right)^{1 / q} \in L^{p \text {, } \Phi}$ with $1<q<\infty$ and the doubling constant $D(\Phi)$ satisfy $1 \leq D(\Phi)<2^{n}$. Then, for any $1 \leq p<\infty$ and any ball $B=B\left(x_{0}, r\right)$, there exists a constant $C>0$ depending only on $p$ and $q$ such that

$$
\begin{gathered}
\left(\int_{\mathbb{R}^{n}}\left(\sum_{j}\left|f_{j}(x)\right|^{q}\right)^{p / q} M\left(\chi_{B}\right)(x) d x\right)^{1 / p} \\
\leq C \cdot \Phi(r)^{1 / p}\left\|\left(\sum_{j}\left|f_{j}\right|^{q}\right)^{1 / q}\right\|_{L^{p, \Phi}} .
\end{gathered}
$$

Proof. Let $\left(\sum_{j}\left|f_{j}\right|^{q}\right)^{1 / q} \in L^{p, \Phi}$ with $1<q<\infty$ and $1 \leq p<$ $\infty$. Then,

$$
\begin{aligned}
& \left(\int_{\mathbb{R}^{n}}\left(\sum_{j}\left|f_{j}(x)\right|^{q}\right)^{p / q} M\left(\chi_{B}\right)(x) d x\right)^{1 / p} \\
& \leq\left(\int_{2 B}\left(\sum_{j}\left|f_{j}(x)\right|^{q}\right)^{p / q} M\left(\chi_{B}\right)(x) d x\right)^{1 / p} \\
& \quad+\left(\int_{(2 B)^{c}}\left(\sum_{j}\left|f_{j}(x)\right|^{q}\right)^{p / q} M\left(\chi_{B}\right)(x) d x\right)^{1 / p} \\
& =I_{1}+I_{2} .
\end{aligned}
$$

By a standard estimate of maximal functions $M\left(\chi_{B}\right)$, we get

$$
\begin{aligned}
I_{1} & \leq\left(\int_{2 B}\left(\sum_{j}\left|f_{j}(x)\right|^{q}\right)^{p / q} d x\right)^{1 / p} \\
& \leq \Phi(2 r)^{1 / p}\left\|\left(\sum_{j}\left|f_{j}\right|^{q}\right)^{1 / q}\right\|_{L^{p, \Phi}} \\
& \leq C \cdot \Phi(r)^{1 / p}\left\|\left(\sum_{j}\left|f_{j}\right|^{q}\right)^{1 / q}\right\|_{L^{p, \Phi}},
\end{aligned}
$$

$$
I_{2} \leq \sum_{\ell=1}^{\infty}\left(\int_{2^{\ell+1} B \backslash 2^{\ell} B}\left(\sum_{j}\left|f_{j}(x)\right|^{q}\right)^{p / q} M\left(\chi_{B}\right)(x) d x\right)^{1 / p}
$$




$$
\begin{aligned}
& \leq C \sum_{\ell=1}^{\infty}\left(\frac{1}{2^{(\ell+1) n}} \int_{2^{\ell+1} B \backslash 2^{\ell} B}\left(\sum_{j}\left|f_{j}(x)\right|^{q}\right)^{p / q} d x\right)^{1 / p} \\
& \leq C \sum_{\ell=1}^{\infty} \frac{\Phi\left(2^{\ell+1} r\right)^{1 / p}}{\left[2^{(\ell+1) n}\right]^{1 / p}}\left\|\left(\sum_{j}\left|f_{j}\right|^{q}\right)^{1 / q}\right\|_{L^{p, \Phi}} \\
& \leq C \cdot \Phi(r)^{1 / p}\left\|\left(\sum_{j}\left|f_{j}\right|^{q}\right)^{1 / q}\right\|_{L^{p, \Phi}},
\end{aligned}
$$

where the last inequality is due to $1 \leq D(\Phi)<2^{n}$. We are done.

Proof of Theorem 18. Let $\left(\sum_{j}\left|f_{j}\right|^{q}\right)^{1 / q} \in L^{p, \Phi}$ with $1<p \leq$ $q<\infty$. We first recall the following vector-valued maximal inequality established by Fefferman and Stein (see [1]); there exists a constant $C>0$ depending on $p, q$, and $n$ such that

$$
\begin{aligned}
& \int_{\mathbb{R}^{n}}\left(\sum_{j}\left|M\left(f_{j}\right)(x)\right|^{q}\right) w(x) d x \\
& \leq \int_{\mathbb{R}^{n}}\left(\sum_{j}\left|f_{j}(y)\right|^{q}\right) M(w)(y) d y
\end{aligned}
$$

holds for any positive functions $f_{1}, f_{2}, \ldots$ and any weight function $w$. Moreover, it can be easily shown using vectorvalued interpolation that, for all $1<p \leq q$ (see $[1,20])$,

$$
\begin{gathered}
\int_{\mathbb{R}^{n}}\left(\sum_{j}\left|M\left(f_{j}\right)(x)\right|^{q}\right)^{p / q} w(x) d x \\
\leq \int_{\mathbb{R}^{n}}\left(\sum_{j}\left|f_{j}(y)\right|^{q}\right)^{p / q} M(w)(y) d y .
\end{gathered}
$$

It is worth pointing out that the above estimate (94) is false in general in the range $p>q$ (see [21]). For any ball $B=$ $B\left(x_{0}, r\right) \subseteq \mathbb{R}^{n}$ with $x_{0} \in \mathbb{R}^{n}$ and $r>0$, we take $w(x)$ as the characteristic function $\left(\chi_{B}\right)(x)$ of the ball $B=B\left(x_{0}, r\right)$; then, by Lemma 23 and (94), we get

$$
\begin{aligned}
& \frac{1}{\Phi(r)^{1 / p}}\left(\int_{B\left(x_{0}, r\right)}\left(\sum_{j}\left|M\left(f_{j}\right)(x)\right|^{q}\right)^{p / q} d x\right)^{1 / p} \\
& \leq \frac{1}{\Phi(r)^{1 / p}}\left(\int_{\mathbb{R}^{n}}\left(\sum_{j}\left|f_{j}(x)\right|^{q}\right)^{p / q} M\left(\chi_{B}\right)(x) d x\right)^{1 / p} \\
& \leq C \cdot \frac{1}{\Phi(r)^{1 / p}} \cdot \Phi(r)^{1 / p}\left\|\left(\sum_{j}\left|f_{j}\right|^{q}\right)^{1 / q}\right\|_{L^{p, \Phi}} \\
& \leq C\left\|\left(\sum_{j}\left|f_{j}\right|^{q}\right)^{1 / q}\right\|_{L^{p, \Phi}} .
\end{aligned}
$$

Therefore, taking the supremum over all balls $B=B\left(x_{0}, r\right) \subseteq$ $\mathbb{R}^{n}$, we complete the proof of Theorem 18 .
Proof of Theorem 19. Let $\left(\sum_{j}\left|f_{j}\right|^{q}\right)^{1 / q} \in L^{1, \Phi}$ with $1<q<\infty$. In [21], Pérez proved that, for each weight $w$ and any positive functions $f_{1}, f_{2}, \ldots$, there exists a constant $C>0$ depending only on $q$ and $n$ such that

$$
\begin{gathered}
w\left(\left\{x \in \mathbb{R}^{n}:\left(\sum_{j}\left|M\left(f_{j}\right)(x)\right|^{q}\right)^{1 / q}>\lambda\right\}\right) \\
\leq \frac{C}{\lambda} \int_{\mathbb{R}^{n}}\left(\sum_{j}\left|f_{j}(y)\right|^{q}\right)^{1 / q} M w(y) d y
\end{gathered}
$$

holds for all $\lambda>0$. For each fixed ball $B=B\left(x_{0}, r\right) \subseteq \mathbb{R}^{n}$, we again take $w(x)$ to be the characteristic function $\left(\chi_{B}\right)(x)$ of the ball $B=B\left(x_{0}, r\right)$; then, by using Lemma 23 and (96), we obtain

$$
\begin{aligned}
& \left|\left\{x \in B:\left(\sum_{j}\left|M\left(f_{j}\right)(x)\right|^{q}\right)^{1 / q}>\lambda\right\}\right| \\
& =\left|\left\{x \in \mathbb{R}^{n}:\left(\sum_{j}\left|M\left(f_{j}\right)(x)\right|^{q}\right)^{1 / q}>\lambda\right\} \cap B\right| \\
& \leq \frac{C}{\lambda} \int_{\mathbb{R}^{n}}\left(\sum_{j}\left|f_{j}(y)\right|^{q}\right)^{1 / q} M\left(\chi_{B}\right)(y) d y \\
& \leq \frac{C \cdot \Phi(r)}{\lambda}\left\|\left(\sum_{j}\left|f_{j}\right|^{q}\right)^{1 / q}\right\|_{L^{1, \Phi}} .
\end{aligned}
$$

Taking the supremum over all balls $B=B\left(x_{0}, r\right) \subseteq \mathbb{R}^{n}$ and all $\lambda>0$, we finish the proof of Theorem 19 .

\subsection{Proofs of Theorems 20, 21, and 22}

Proof of Theorems 20 and 22. Let $\left(\sum_{j}\left|f_{j}\right|^{q}\right)^{1 / q} \in L^{p, \Phi}$ with $1<p, q<\infty$. For any ball $B=B\left(x_{0}, r\right) \subseteq \mathbb{R}^{n}$ with $x_{0} \in \mathbb{R}^{n}$ and $r>0$, we write $f_{j}=f_{j}^{0}+f_{j}^{\infty}$, where $f_{j}^{0}=f_{j} \cdot \chi_{2 B}$, $j=1,2, \ldots$. Then, we have

$$
\begin{aligned}
& \frac{1}{\Phi(r)^{1 / p}}\left(\int_{B\left(x_{0}, r\right)}\left(\sum_{j}\left|T_{\Omega}\left(f_{j}\right)(x)\right|^{q}\right)^{p / q} d x\right)^{1 / p} \\
& \leq \frac{1}{\Phi(r)^{1 / p}}\left(\int_{B\left(x_{0}, r\right)}\left(\sum_{j}\left|T_{\Omega}\left(f_{j}^{0}\right)(x)\right|^{q}\right)^{p / q} d x\right)^{1 / p} \\
& \quad+\frac{1}{\Phi(r)^{1 / p}}\left(\int_{B\left(x_{0}, r\right)}\left(\sum_{j}\left|T_{\Omega}\left(f_{j}^{\infty}\right)(x)\right|^{q}\right)^{p / q} d x\right)^{1 / p} \\
& =J_{1}+J_{2} .
\end{aligned}
$$


International Journal of Mathematics and Mathematical Sciences

13

Applying Theorems 9 and 11 and the doubling condition (33), we obtain

$$
\begin{aligned}
J_{1} & \leq \frac{1}{\Phi(r)^{1 / p}}\left\|\left(\sum_{j}\left|T_{\Omega}\left(f_{j}^{0}\right)\right|^{q}\right)^{1 / q}\right\|_{L^{p}} \\
& \leq C \cdot \frac{1}{\Phi(r)^{1 / p}}\left(\int_{2 B}\left(\sum_{j}\left|f_{j}(x)\right|^{q}\right)^{p / q} d x\right)^{1 / p} \\
& \leq C\left\|\left(\sum_{j}\left|f_{j}\right|^{q}\right)^{1 / q}\right\|_{L^{p, \Phi}} \cdot \frac{\Phi(2 r)^{1 / p}}{\Phi(r)^{1 / p}} \\
& \leq C\left\|\left(\sum_{j}\left|f_{j}\right|^{q}\right)^{1 / q}\right\|_{p^{p, \Phi}} .
\end{aligned}
$$

Let us analyze the second term $J_{2}$. To this end, we will consider the following two cases. (i) When $\Omega \in L^{\infty}\left(S^{n-1}\right)$, we use the estimate (68) along with Hölder's inequality to obtain

$$
\begin{aligned}
& \left(\sum_{j}\left|T_{\Omega}\left(f_{j}^{\infty}\right)(x)\right|^{q}\right)^{1 / q} \\
& \leq C \sum_{\ell=1}^{\infty} \frac{1}{2^{\ell+1} B \mid} \int_{2^{\ell+1} B}\left(\sum_{j}\left|f_{j}(y)\right|^{q}\right)^{1 / q} d y \\
& \leq C \sum_{\ell=1}^{\infty} \frac{1}{2^{\ell+1} B \mid}\left(\int_{2^{\ell+1} B}\left(\sum_{j}\left|f_{j}(x)\right|^{q}\right)^{p / q} d x\right)^{1 / p} \\
& \quad \times\left(\int_{2^{\ell+1} B} 1 d x\right)^{1 / p^{\prime}} \\
& \leq C\left\|\left(\sum_{j}\left|f_{j}\right|^{q}\right)^{1 / q}\right\|_{L^{p, \Phi}} \cdot \sum_{\ell=1}^{\infty} \frac{\Phi\left(2^{\ell+1} r\right)^{1 / p}}{\left|B\left(x_{0}, 2^{\ell+1} r\right)\right|^{1 / p}} .
\end{aligned}
$$

(ii) The second case is when $\Omega \in L^{s}\left(S^{n-1}\right)$ with $1<s<\infty$. In the proof of Theorem 17, we have already proved that, for any $x \in B$ (see (86)),

$$
\begin{aligned}
& \left(\sum_{j}\left|T_{\Omega}\left(f_{j}^{\infty}\right)(x)\right|^{q}\right)^{1 / q} \\
& \leq C \sum_{\ell=1}^{\infty}\left(\frac{1}{\left|2^{\ell+1} B\right|} \int_{2^{\ell+1} B}\left(\sum_{j}\left|f_{j}(y)\right|^{q}\right)^{s^{\prime} / q} d y\right)^{1 / s^{\prime}} .
\end{aligned}
$$

For the case of $p=s^{\prime}$, we have

$$
\begin{aligned}
& \left(\sum_{j}\left|T_{\Omega}\left(f_{j}^{\infty}\right)(x)\right|^{q}\right)^{1 / q} \\
& \leq C \sum_{\ell=1}^{\infty}\left(\frac{1}{\left|2^{\ell+1} B\right|} \int_{2^{\ell+1} B}\left(\sum_{j}\left|f_{j}(y)\right|^{q}\right)^{p / q} d y\right)^{1 / p} \\
& \leq C\left\|\left(\sum_{j}\left|f_{j}\right|^{q}\right)^{1 / q}\right\|_{L^{p, \Phi}} \cdot \sum_{\ell=1}^{\infty} \frac{\Phi\left(2^{\ell+1} r\right)^{1 / p}}{\left|B\left(x_{0}, 2^{\ell+1} r\right)\right|^{1 / p}} .
\end{aligned}
$$

We now proceed to the case where $p>s^{\prime}$; in the present situation, we also set $t=p / s^{\prime}>1$. Then, by using Hölder's inequality with exponent $t>1$, we deduce that

$$
\begin{aligned}
& \left(\sum_{j}\left|T_{\Omega}\left(f_{j}^{\infty}\right)(x)\right|^{q}\right)^{1 / q} \\
& \leq C \sum_{\ell=1}^{\infty} \frac{1}{\left|2^{\ell+1} B\right|^{1 / s^{\prime}}}\left(\int_{2^{\ell+1} B}\left(\sum_{j}\left|f_{j}(y)\right|^{q}\right)^{p / q} d y\right)^{1 / p} \\
& \quad \times\left(\int_{2^{\ell+1} B} 1 d y\right)^{1 / t^{\prime} s^{\prime}} \\
& \leq C \sum_{\ell=1}^{\infty}\left(\frac{1}{\left|2^{\ell+1} B\right|} \int_{2^{\ell+1} B}\left(\sum_{j}\left|f_{j}(y)\right|^{q}\right)^{p / q} d y\right)^{1 / p} \\
& \leq C \mid\left(\sum_{j}\left|f_{j}\right|^{q}\right)^{1 / q}\|\|_{L^{p, \Phi}} \cdot \sum_{\ell=1}^{\infty} \frac{\Phi\left(2^{\ell+1} r\right)^{1 / p}}{\left|B\left(x_{0}, 2^{\ell+1} r\right)\right|^{1 / p}} .
\end{aligned}
$$

Summarizing the estimates (100)-(103) derived above, we conclude that

$$
\begin{aligned}
J_{2} \leq C & \left\|\left(\sum_{j}\left|f_{j}\right|^{q}\right)^{1 / q}\right\|_{L^{p, \Phi}} \\
& \cdot \sum_{\ell=1}^{\infty} \frac{\left|B\left(x_{0}, r\right)\right|^{1 / p}}{\Phi(r)^{1 / p}} \cdot \frac{\Phi\left(2^{\ell+1} r\right)^{1 / p}}{\left|B\left(x_{0}, 2^{\ell+1} r\right)\right|^{1 / p}} .
\end{aligned}
$$

Since $1 \leq D(\Phi)<2^{n}$, then by using the doubling condition (33) of $\Phi$, we know that the above series is bounded by an absolute constant:

$$
\begin{aligned}
& \sum_{\ell=1}^{\infty} \frac{\left|B\left(x_{0}, r\right)\right|^{1 / p}}{\Phi(r)^{1 / p}} \cdot \frac{\Phi\left(2^{\ell+1} r\right)^{1 / p}}{\left|B\left(x_{0}, 2^{\ell+1} r\right)\right|^{1 / p}} \\
& \leq C \sum_{\ell=1}^{\infty}\left(\frac{D(\Phi)}{2^{n}}\right)^{(\ell+1) / p} \\
& \leq C .
\end{aligned}
$$


Therefore,

$$
J_{2} \leq C\left\|\left(\sum_{j}\left|f_{j}\right|^{q}\right)^{1 / q}\right\|_{L^{p, \Phi}} .
$$

Combining the above estimates for $J_{1}$ and $J_{2}$ and then taking the supremum over all balls $B=B\left(x_{0}, r\right) \subseteq \mathbb{R}^{n}$, we complete the proof of Theorems 20 and 22.

Proof of Theorem 21. Let $\left(\sum_{j}\left|f_{j}\right|^{q}\right)^{1 / q} \in L^{1, \Phi}$ with $1<q<\infty$. For each fixed ball $B=B\left(x_{0}, r\right) \subseteq \mathbb{R}^{n}$, we again decompose $f_{j}$ as $f_{j}=f_{j}^{0}+f_{j}^{\infty}$, where $f_{j}^{0}=f_{j} \cdot \chi_{2 B}, j=1,2, \ldots$ For any given $\lambda>0$, we write

$$
\begin{aligned}
& \left|\left\{x \in B:\left(\sum_{j}\left|T_{\Omega}\left(f_{j}\right)(x)\right|^{q}\right)^{1 / q}>\lambda\right\}\right| \\
& \leq\left|\left\{x \in B:\left(\sum_{j}\left|T_{\Omega}\left(f_{j}^{0}\right)(x)\right|^{q}\right)^{1 / q}>\frac{\lambda}{2}\right\}\right| \\
& \quad+\left|\left\{x \in B:\left(\sum_{j}\left|T_{\Omega}\left(f_{j}^{\infty}\right)(x)\right|^{q}\right)^{1 / q}>\frac{\lambda}{2}\right\}\right| \\
& =J_{1}^{\prime}+J_{2}^{\prime} .
\end{aligned}
$$

Let us start with the term $J_{1}^{\prime}$. Theorem 10 and the doubling condition (33) imply that

$$
\begin{aligned}
J_{1}^{\prime} & \leq \frac{2}{\lambda} \cdot\left\|\left(\sum_{j}\left|T_{\Omega}\left(f_{j}^{0}\right)\right|^{q}\right)^{1 / q}\right\|_{W L^{1}} \\
& \leq \frac{C}{\lambda} \cdot\left(\int_{2 B}\left(\sum_{j}\left|f_{j}(x)\right|^{q}\right)^{1 / q} d x\right) \\
& \leq \frac{C \cdot \Phi(2 r)}{\lambda}\left\|\left(\sum_{j}\left|f_{j}\right|^{q}\right)^{1 / q}\right\|_{L^{1, \Phi}} \\
& \leq \frac{C \cdot \Phi(r)}{\lambda}\left\|\left(\sum_{j}\left|f_{j}\right|^{q}\right)^{1 / q}\right\|_{L^{1, \Phi}} .
\end{aligned}
$$

We turn our attention to the estimate of $J_{2}^{\prime}$. It follows directly from the estimate (68) that, for any $x \in B$,

$$
\begin{aligned}
& \left(\sum_{j}\left|T_{\Omega}\left(f_{j}^{\infty}\right)(x)\right|^{q}\right)^{1 / q} \\
& \leq C \sum_{\ell=1}^{\infty} \frac{1}{2^{\ell+1} B \mid} \int_{2^{\ell+1} B}\left(\sum_{j}\left|f_{j}(y)\right|^{q}\right)^{1 / q} d y \\
& \leq C\left\|\left(\sum_{j}\left|f_{j}\right|^{q}\right)^{1 / q}\right\|_{L^{1, \Phi}} \cdot \sum_{\ell=1}^{\infty} \frac{\Phi\left(2^{\ell+1} r\right)}{\left|B\left(x_{0}, 2^{\ell+1} r\right)\right|}
\end{aligned}
$$

$$
\begin{gathered}
\leq C\left\|\left(\sum_{j}\left|f_{j}\right|^{q}\right)^{1 / q}\right\|_{L^{1, \Phi}} \cdot \frac{\Phi(r)}{\left|B\left(x_{0}, r\right)\right|} \\
\times \sum_{\ell=1}^{\infty} \frac{\left|B\left(x_{0}, r\right)\right|}{\Phi(r)} \cdot \frac{\Phi\left(2^{\ell+1} r\right)}{\left|B\left(x_{0}, 2^{\ell+1} r\right)\right|} .
\end{gathered}
$$

Notice that $1 \leq D(\Phi)<2^{n}$. Arguing as in the proof of (105), we can get

$$
\sum_{\ell=1}^{\infty} \frac{\left|B\left(x_{0}, r\right)\right|}{\Phi(r)} \cdot \frac{\Phi\left(2^{\ell+1} r\right)}{\left|B\left(x_{0}, 2^{\ell+1} r\right)\right|} \leq \sum_{\ell=1}^{\infty}\left(\frac{D(\Phi)}{2^{n}}\right)^{\ell+1} \leq C .
$$

Hence, for any $x \in B\left(x_{0}, r\right)$,

$$
\left(\sum_{j}\left|T_{\Omega}\left(f_{j}^{\infty}\right)(x)\right|^{q}\right)^{1 / q} \leq C\left\|\left(\sum_{j}\left|f_{j}\right|^{q}\right)^{1 / q}\right\|_{L^{1, \Phi}} \cdot \frac{\Phi(r)}{\left|B\left(x_{0}, r\right)\right|} .
$$

If $\left\{x \in B:\left(\sum_{j}\left|T_{\Omega}\left(f_{j}^{\infty}\right)(x)\right|^{q}\right)^{1 / q}>\lambda / 2\right\}=\emptyset$, then the inequality

$$
J_{2}^{\prime} \leq \frac{C \cdot \Phi(r)}{\lambda}\left\|\left(\sum_{j}\left|f_{j}\right|^{q}\right)^{1 / q}\right\|_{L^{1, \Phi}}
$$

holds trivially. Now if instead we suppose that

$$
\left\{x \in B:\left(\sum_{j}\left|T_{\Omega}\left(f_{j}^{\infty}\right)(x)\right|^{q}\right)^{1 / q}>\frac{\lambda}{2}\right\} \neq \emptyset,
$$

then, by the pointwise inequality (111), we can see that

$$
\lambda \leq C\left\|\left(\sum_{j}\left|f_{j}\right|^{q}\right)^{1 / q}\right\|_{L^{1, \Phi}} \cdot \frac{\Phi(r)}{\left|B\left(x_{0}, r\right)\right|},
$$

which in turn gives us that

$$
\left|B\left(x_{0}, r\right)\right| \leq \frac{C \cdot \Phi(r)}{\lambda}\left\|\left(\sum_{j}\left|f_{j}\right|^{q}\right)^{1 / q}\right\|_{L^{1, \Phi}} .
$$

Therefore,

$$
J_{2}^{\prime} \leq\left|B\left(x_{0}, r\right)\right| \leq \frac{C \cdot \Phi(r)}{\lambda}\left\|\left(\sum_{j}\left|f_{j}\right|^{q}\right)^{1 / q}\right\|_{L^{1, \Phi}} .
$$

Summing up the above estimates for $J_{1}^{\prime}$ and $J_{2}^{\prime}$ and then taking the supremum over all balls $B=B\left(x_{0}, r\right) \subseteq \mathbb{R}^{n}$ and all $\lambda>0$, we conclude the proof of Theorem 21.

\section{Conflict of Interests}

The author declares that there is no conflict of interests regarding the publication of this paper. 


\section{References}

[1] C. Fefferman and E. M. Stein, "Some maximal inequalities," American Journal of Mathematics, vol. 93, pp. 107-115, 1971.

[2] J. García-Cuerva and J. L. Rubio de Francia, Weighted Norm Inequalities and Related Topics, vol. 116, North-Holland, Amsterdam, The Netherlands, 1985.

[3] B. Muckenhoupt, "Weighted norm inequalities for the Hardy maximal function," Transactions of the American Mathematical Society, vol. 165, pp. 207-226, 1972.

[4] K. F. Andersen and R. T. John, "Weighted inequalities for vector-valued maximal functions and singular integrals," Studia Mathematica, vol. 69, no. 1, pp. 19-31, 1980.

[5] C. L. Wu, "A characterization of some weighted inequalities for the vector-valued weighted maximal function," Acta Mathematica Sinica, vol. 26, no. 11, pp. 2191-2198, 2010.

[6] C. Pérez and R. Trujillo-González, "Sharp weighted estimates for vector-valued singular integral operators and commutators," The Tohoku Mathematical Journal, vol. 55, no. 1, pp. 109-129, 2003.

[7] J. L. Rubio de Francia, F. J. Ruiz, and J. L. Torrea, "CalderónZygmund theory for operator-valued kernels," Advances in Mathematics, vol. 62, no. 1, pp. 7-48, 1986.

[8] A. Benedek, A. P. Calderón, and R. Panzone, "Convolution operators on Banach space valued functions," Proceedings of the National Academy of Sciences of the United States of America, vol. 48, pp. 356-365, 1962.

[9] C. B. Morrey Jr., "On the solutions of quasi-linear elliptic partial differential equations," Transactions of the American Mathematical Society, vol. 43, no. 1, pp. 126-166, 1938.

[10] D. R. Adams, "A note on Riesz potentials," Duke Mathematical Journal, vol. 42, no. 4, pp. 765-778, 1975.

[11] F. Chiarenza and M. Frasca, "Morrey spaces and HardyLittlewood maximal function," Rendiconti di Matematica e delle sue Applicazioni, vol. 7, no. 3-4, pp. 273-279, 1987.

[12] J. Peetre, "On the theory of $\mathscr{L}_{p, \lambda}$ spaces," Journal of Functional Analysis, vol. 4, no. 1, pp. 71-87, 1969.

[13] T. Mizuhara, "Boundedness of some classical operators on generalized Morrey spaces," in Harmonic Analysis, ICM-90 Satellite Conference Proceedings, pp. 183-189, Springer, 1991.

[14] V. S. Guliyev, "Boundedness of the maximal, potential and singular operators in the generalized Morrey spaces," Journal of Inequalities and Applications, vol. 2009, Article ID 503948, 20 pages, 2009.

[15] V. S. Guliyev, S. S. Aliyev, and T. Karaman, "Boundedness of a class of sublinear operators and their commutators on generalized Morrey spaces," Abstract and Applied Analysis, vol. 2011, Article ID 356041, 18 pages, 2011.

[16] V. S. Guliyev, S. S. Aliyev, T. Karaman, and P. S. Shukurov, "Boundedness of sublinear operators and commutators on generalized Morrey spaces," Integral Equations and Operator Theory, vol. 71, no. 3, pp. 327-355, 2011.

[17] S. Lu, D. Yang, and Z. Zhou, "Sublinear operators with rough kernel on generalized Morrey spaces," Hokkaido Mathematical Journal, vol. 27, no. 1, pp. 219-232, 1998.

[18] E. Nakai, "Hardy-Littlewood maximal operator, singular integral operators and the Riesz potentials on generalized Morrey spaces," Mathematische Nachrichten, vol. 166, pp. 95-103, 1994.

[19] Y. Komori and S. Shirai, "Weighted Morrey spaces and a singular integral operator," Mathematische Nachrichten, vol. 282, no. 2, pp. 219-231, 2009.
[20] D. Cruz-Uribe and C. Pérez, "Two weight extrapolation via the maximal operator," Journal of Functional Analysis, vol. 174, no. 1, pp. 1-17, 2000.

[21] C. Pérez, "Sharp weighted inequalities for the vector-valued maximal function," Transactions of the American Mathematical Society, vol. 352, no. 7, pp. 3265-3288, 2000. 


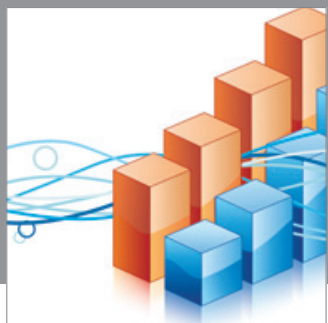

Advances in

Operations Research

mansans

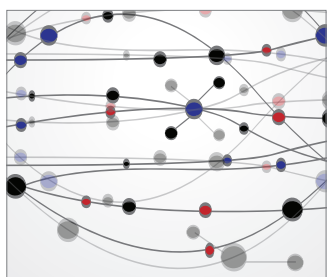

The Scientific World Journal
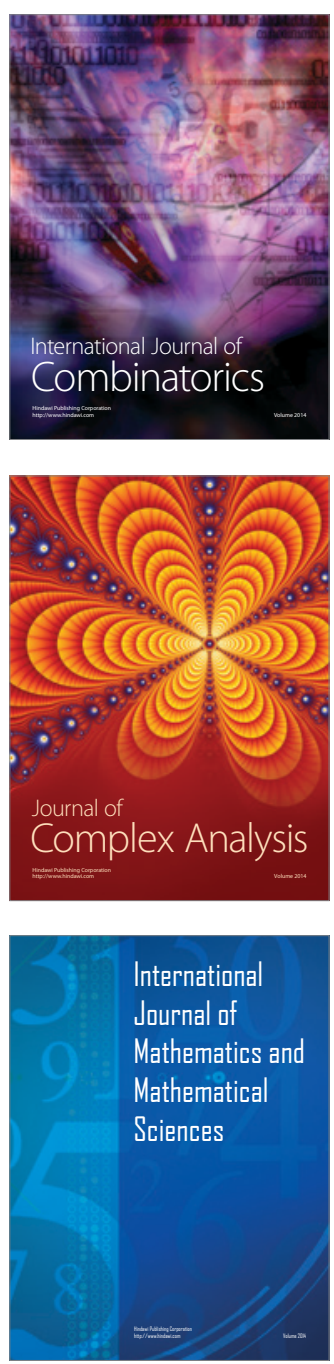
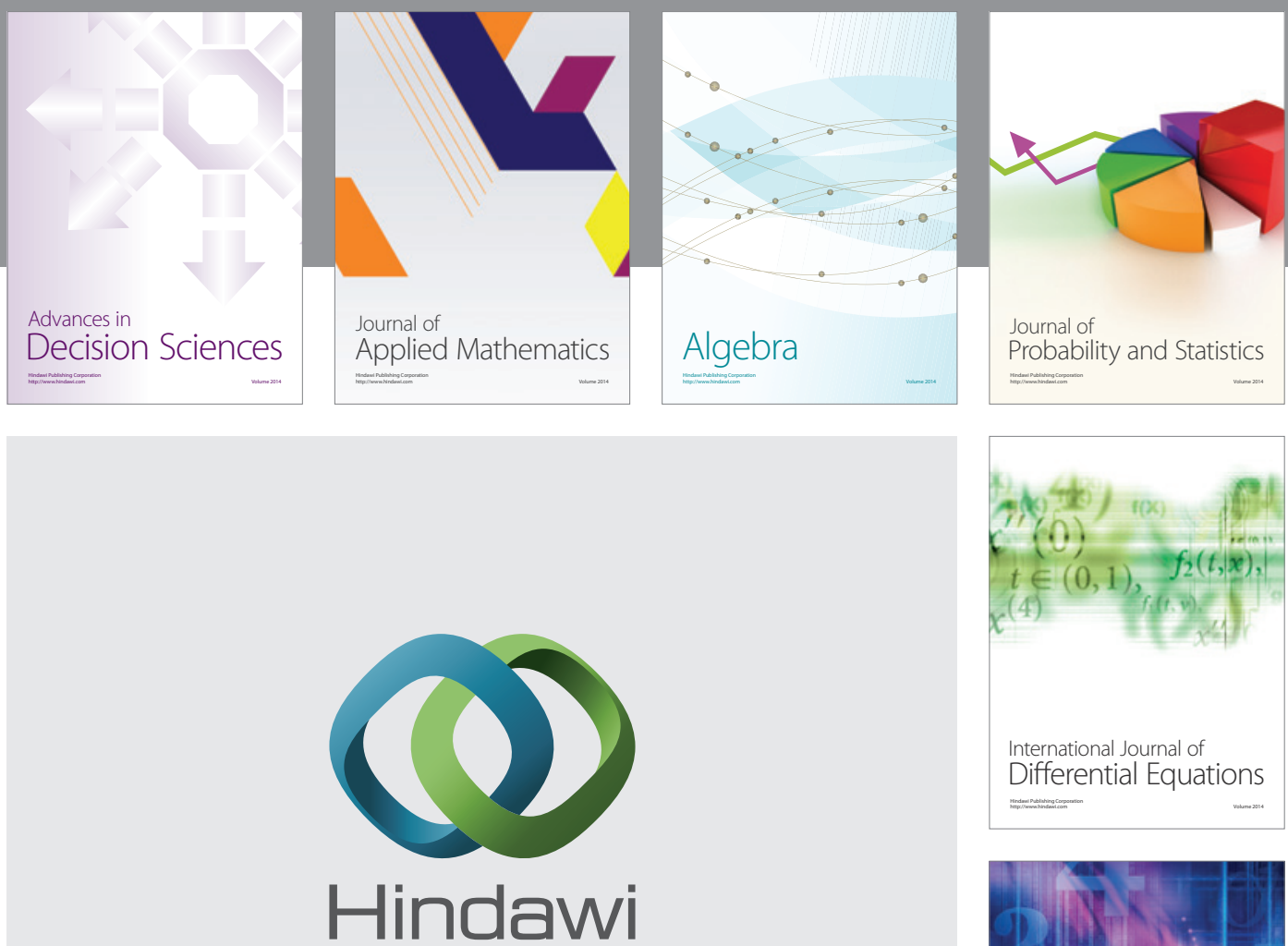

Submit your manuscripts at http://www.hindawi.com
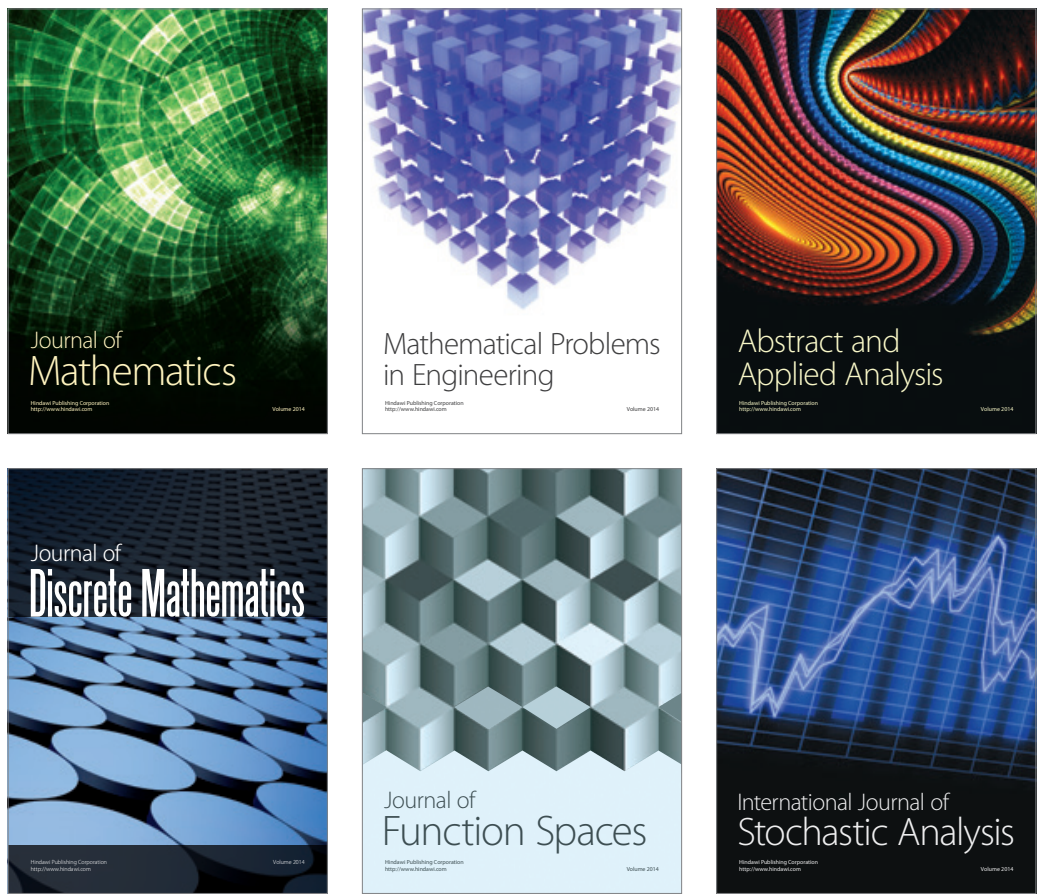

Journal of

Function Spaces

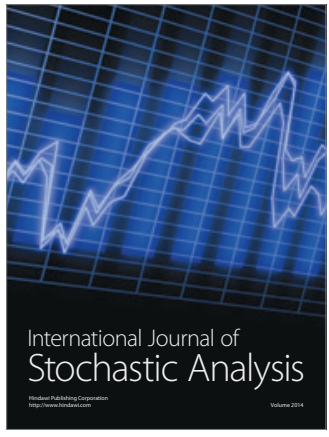

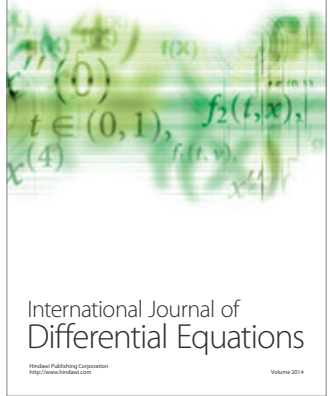
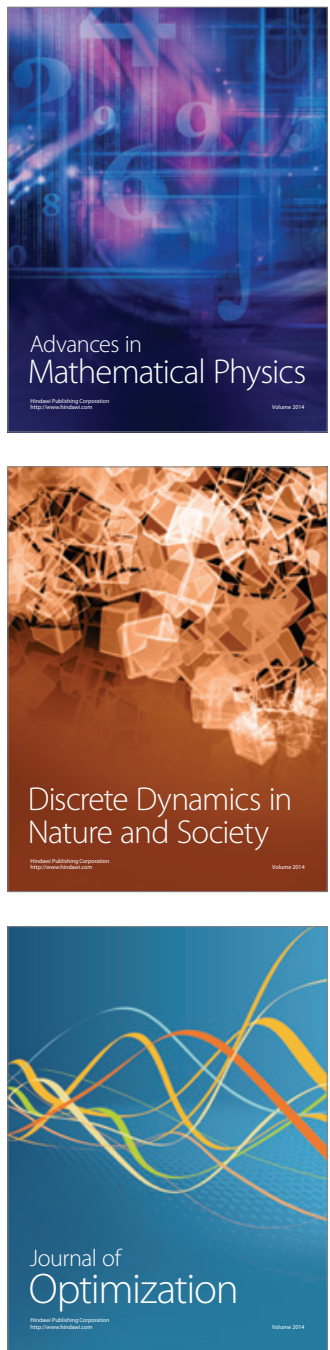